\title{
FRIEZE PATTERNS WITH COEFFICIENTS
}

\author{
MICHAEL CUNTZ ${ }^{1}$, THORSTEN HOLM ${ }^{1}$ and PETER JØRGENSEN ${ }^{2}$ \\ ${ }^{1}$ Leibniz Universität Hannover, Institut für Algebra, Zahlentheorie und Diskrete Mathematik, \\ Fakultät für Mathematik und Physik, Welfengarten 1, D-30167 Hannover, Germany; \\ email: cuntz@math.uni-hannover.de,holm@math.uni-hannover.de \\ ${ }^{2}$ School of Mathematics and Statistics, Newcastle University, \\ Newcastle upon Tyne NE1 7RU, UK; \\ email: peter.jorgensen@ncl.ac.uk
}

Received 16 September 2019; accepted 27 February 2020

\begin{abstract}
Frieze patterns, as introduced by Coxeter in the 1970s, are closely related to cluster algebras without coefficients. A suitable generalization of frieze patterns, linked to cluster algebras with coefficients, has only briefly appeared in an unpublished manuscript by Propp. In this paper, we study these frieze patterns with coefficients systematically and prove various fundamental results, generalizing classic results for frieze patterns. As a consequence, we see how frieze patterns with coefficients can be obtained from classic frieze patterns by cutting out subpolygons from the triangulated polygons associated with classic Conway-Coxeter frieze patterns. We address the question of which frieze patterns with coefficients can be obtained in this way and solve this problem completely for triangles. Finally, we prove a finiteness result for frieze patterns with coefficients by showing that for a given boundary sequence there are only finitely many (nonzero) frieze patterns with coefficients with entries in a subset of the complex numbers without an accumulation point.
\end{abstract}

2010 Mathematics Subject Classification: 13F60 (primary); 05E15, 05E99, 51M20 (secondary)

\section{Introduction}

Frieze patterns were introduced by Coxeter [4], and a beautiful theory for frieze patterns over positive integers was developed subsequently by Conway and Coxeter [3]. Only some three decades later, the importance of frieze patterns for other areas of mathematics became clearer when Fomin and Zelevinsky invented cluster algebras [8]. Namely, the exchange condition in cluster algebras mimics the local condition defining frieze patterns. In this way, starting with a

(c) The Author(s) 2020. This is an Open Access article, distributed under the terms of the Creative Commons Attribution licence (http://creativecommons.org/licenses/by/4.0/), which permits unrestricted re-use, distribution, and reproduction in any medium, provided the original work is properly cited. 
set of indeterminates, the entries in a frieze pattern (over the field of rational functions) are precisely the cluster variables of the corresponding cluster algebra of Dynkin type $A$ with trivial coefficients. Via cluster algebras, frieze patterns are now connected to many areas of mathematics and therefore, frieze patterns are currently a very active area of research; see the survey by Morier-Genoud [11] for more details.

Classically, a frieze pattern is an infinite array of the form

$$
\begin{array}{ccccccccccc}
0 & 1 & c_{i-1, i+1} & c_{i-1, i+2} & \ldots & \ldots & c_{i-1, n+i} & 1 & 0 & & \\
& 0 & 1 & c_{i, i+2} & c_{i, i+3} & \ldots & \ldots & c_{i, n+i+1} & 1 & 0 & \\
& 0 & 1 & c_{i+1, i+3} & c_{i+1, i+4} & \ldots & \ldots & c_{i+1, n+i+2} & 1 & 0 \\
& & & \ddots & & & & \ddots & &
\end{array}
$$

such that all neighbouring $2 \times 2$-matrices have determinant 1 .

From the cluster algebra viewpoint, the two bounding diagonals of 1's mean to set all the coefficients (or frozen variables) in the cluster algebra equal to 1 . Basically, the entire extensive recent literature on frieze patterns deals with such classic frieze patterns.

In the theory of cluster algebras the coefficients are very important, so it would be natural to consider more general frieze patterns where the bounding diagonals can have arbitrary entries. Such frieze patterns have appeared briefly already in an unpublished manuscript by Propp [13]. In particular, one finds the modified local condition on $2 \times 2$-determinants, now involving the boundary entries. This is set up in such a way that, again, the entries in a certain frieze pattern (over the field of rational functions) are precisely the cluster variables of the corresponding cluster algebra with coefficients.

Since cluster algebras (with coefficients) are linked to numerous areas in mathematics, the corresponding more general frieze patterns including the coefficients have implicitly appeared in the literature, for example in the context of $T$-systems, the octahedron recurrence [15] or as Plücker friezes [1], but so far they have not been systematically studied. One aim of the present paper is to develop the general theory of these more general frieze patterns, which we call frieze patterns with coefficients; see Definition 2.1 for a precise definition. As in the classic case, a satisfactory theory can only be expected for tame frieze patterns with coefficients, which imposes that all adjacent $3 \times 3$-determinants are 0 . (There are far too many wild frieze patterns: see [5].)

In Sections 2 and 3, we develop a theory for tame frieze patterns with coefficients, generalizing several of the well-known results for classic frieze patterns. 
We introduce certain $2 \times 2$-matrices, called $\mu$-matrices, which govern the propagation along the rows and the columns of a frieze pattern with coefficients, that is, multiplication with such matrices transforms two consecutive entries in a row or a column to the next two consecutive entries. See Definition 2.8 and Proposition 2.10. Moreover, we present in Corollary 2.11 formulae for how every entry in the frieze pattern appears in a certain product of $\mu$-matrices.

As another application of the propagation formulae, we prove that every tame frieze pattern with coefficients satisfies a glide reflection; see Theorem 2.12. A fundamental domain for the entries of the tame frieze pattern with coefficients under the glide reflection can be indexed in such a way that it corresponds bijectively to the edges and diagonals of a regular $(n+3)$-gon (where $n$ is the height of the frieze pattern).

This is the starting point in Section 3. It allows a useful alternative viewpoint for tame frieze patterns with coefficients: the entries in such a frieze pattern become the labels for the edges and diagonals of a polygon (where the boundary entries become the labels for the edges). We show in Theorem 3.3 that these labels have to satisfy many more than the defining local conditions, namely the so-called Ptolemy relations.

For distinguishing the two viewpoints, we speak of a tame frieze with coefficients if we consider a map from the edges and diagonals of a polygon satisfying all Ptolemy relations.

In Section 4, we present a construction for obtaining friezes with coefficients from classic friezes: given a classic frieze, one can cut out any subpolygon and get a frieze with coefficients. If this frieze with coefficients is a classic frieze, then it is a summand in the sense of [6] or [10].

This naturally leads to the question which friezes with coefficients can be obtained from classic friezes by cutting out subpolygons. Small examples already show that not every frieze with coefficients can be obtained from classic friezes in this way.

The rest of Section 4 and also Section 5 deal with this fundamental question for friezes with coefficients over positive integers. Our results not only give insight into friezes with coefficients but also shed new light on the classic ConwayCoxeter friezes, that is, friezes over positive integers with all boundary entries equal to 1 .

In Lemma 4.3, we show that any triangle cut out of a classic Conway-Coxeter frieze has the property that the greatest common divisor of any two of the labels on the triangle divides the third label. Conversely, we show in Lemma 4.5 that given two coprime natural numbers $a, b$, there exists a classic Conway-Coxeter frieze containing a triangle with labels $1, a, b$. The proof of this result exhibits a close connection between friezes and the Euclidean algorithm, which we think might be of interest in its own right. 
Note that the results just mentioned do not completely settle the question which labelled triangles can actually appear as subpolygons of classic Conway-Coxeter friezes. It turns out that this problem is rather subtle, and we address it in Section 5. As a main result we give a complete classification of the problem, that is, we describe the triples $(a, b, c) \in \mathbb{N}^{3}$ such that there exists a triangle in some classic Conway-Coxeter frieze with labels $a, b, c$.

THEOREM 1.1 (Theorem 5.12). Let $a, b, c \in \mathbb{N}$. Then the triple $(a, b, c)$ appears as labels of a triangle in a classic Conway-Coxeter frieze if and only if $\operatorname{gcd}(a, b)=\operatorname{gcd}(b, c)=\operatorname{gcd}(a, c)$ and

$$
v_{2}(a)=v_{2}(b)=v_{2}(c)=0 \quad \text { or } \quad\left|\left\{v_{2}(a), v_{2}(b), v_{2}(c)\right\}\right|>1,
$$

where $v_{2}(n)$ is the 2-valuation of $n$.

In Section 6, we go back to frieze patterns with coefficients having entries in any subset $R \subseteq \mathbb{C}$ (not necessarily integers as in the previous section). We address another fundamental question: For a given boundary sequence, how many frieze patterns with coefficients exist over $R$ ? Our main interest is whether there are finitely or infinitely many such frieze patterns. Easy examples show that allowing entries to be 0 rather quickly leads to infinitely many frieze patterns. So we restrict to frieze patterns with nonzero entries.

In Lemma 6.1, we prove a rather general finiteness result, which for a given boundary sequence yields an upper bound for the values in the quiddity cycle of the corresponding frieze pattern. This generalizes a similar result for classic frieze patterns from [7, Theorem 3.6]. As a consequence, we can conclude that for every subset $R$ of the complex numbers without an accumulation point and every boundary sequence, there exist only finitely many frieze patterns over $R \backslash\{0\}$ with the given boundary sequence; see Proposition 6.3.

\section{Definition and fundamental properties}

In this section, we introduce frieze patterns with coefficients. This concept goes back to an unpublished manuscript by Propp [13]. A large amount of research has been carried out on classic frieze patterns, where all coefficients have been set to 1 . A systematic treatment of the far more general frieze patterns with coefficients does not seem to be present in the literature so far. Therefore, we give a detailed account here, in particular, proving several fundamental properties of such frieze patterns with coefficients, generalizing well-known properties of the classic frieze patterns. 
Definition 2.1. Let $R \subseteq \mathbb{C}$ be a subset of complex numbers. Let $n \in \mathbb{Z}_{\geqslant 0}$.

A frieze pattern with coefficients of height $n$ over $R$ is an infinite array of the form

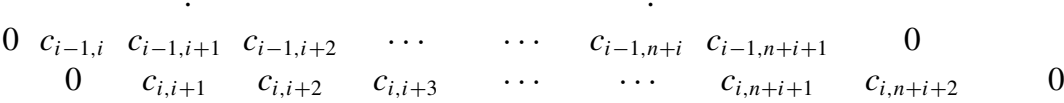

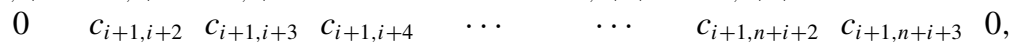

where we also set $c_{i, i}=0=c_{i, n+i+3}$ for all $i \in \mathbb{Z}$, such that the following holds:

(i) $c_{i, j} \in R$ for all $i \in \mathbb{Z}$ and $i<j<n+i+3$.

(ii) $c_{i, i+1} \neq 0$ for all $i \in \mathbb{Z}$.

(iii) For every (complete) adjacent $2 \times 2$-submatrix $\left(\begin{array}{cc}c_{i, j} & c_{i, j+1} \\ c_{i+1, j} & c_{i+1, j+1}\end{array}\right)$, we have

$$
c_{i, j} c_{i+1, j+1}-c_{i, j+1} c_{i+1, j}=c_{i+1, n+i+3} c_{j, j+1} .
$$

REMARK 2.2.

(1) The diagonals with entries $c_{i, i+1}$ and $c_{i, n+i+2}$ are called the boundary of the frieze pattern with coefficients. Part (iii) of the definition says that the determinant of every (complete) adjacent $2 \times 2$-submatrix is given by the product of two specific entries on the boundary of the frieze pattern, namely the numbers in solid boxes in Figure 1.

(2) Note that for $j=i+1$, Equation $\left(E_{i, i+1}\right)$ imposes that

$$
c_{i, i+1} c_{i+1, i+2}=c_{i+1, n+i+3} c_{i+1, i+2} .
$$

Since $c_{i+1, i+2} \neq 0$ by part (ii) and $R \subseteq \mathbb{C}$ has no zero divisors, we conclude that

$$
c_{i, i+1}=c_{i+1, n+i+3} \quad \text { for all } i \in \mathbb{Z} .
$$

Similarly, from Equation $\left(E_{i+1, n+i+3}\right)$ we get that

$$
c_{i+1, n+i+3}=c_{n+i+3, n+i+4} \quad \text { for all } i \in \mathbb{Z} .
$$

This means that there is a glide symmetry on the boundary entries. In particular, applying (1) and (2) we get $c_{i, i+1}=c_{n+i+3, n+i+4}$ for all $i \in \mathbb{Z}$. That is, for a frieze pattern with coefficients of height $n$ the boundary is periodic of period $n+3$. Hence, the entire boundary is determined by a sequence of $n+3$ consecutive boundary entries $\left(c_{i, i+1}, \ldots, c_{n+i+2, n+i+3}\right)$. 


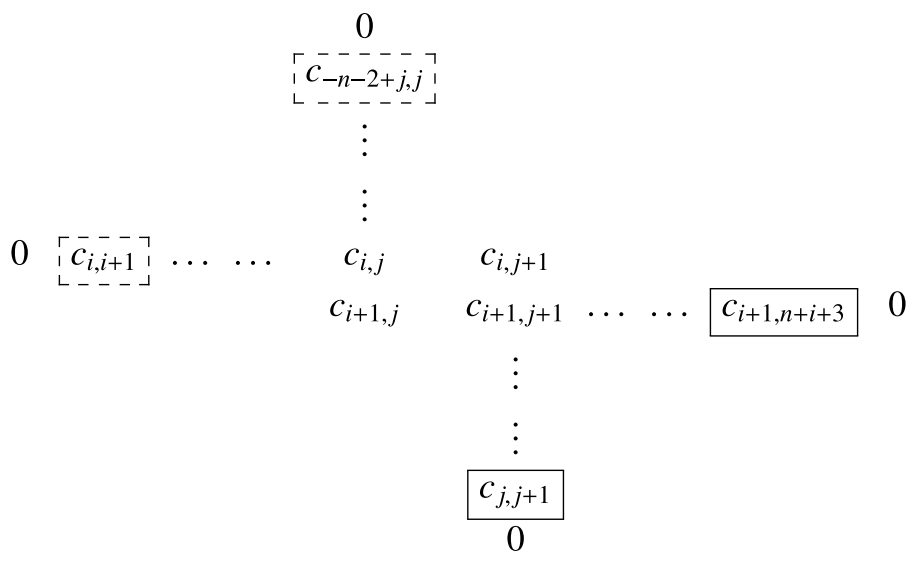

Figure 1. The local condition in a frieze pattern with coefficients.

(3) From the glide symmetry in (1), it also follows that the local condition $\left(E_{i, j}\right)$ in Definition 2.1 could as well be stated in terms of two other boundary entries, namely the numbers in dashed boxes instead of the numbers in solid boxes in Figure 1.

REMARK 2.3. Frieze patterns with coefficients can be scaled, that is, if $\mathcal{C}=\left(c_{i, j}\right)$ is a frieze pattern with coefficients and $z \in \mathbb{C} \backslash\{0\}$, then $z \mathcal{C}:=\left(z c_{i, j}\right)$ is again a frieze pattern with coefficients (possibly over some other subset $R \subseteq \mathbb{C}$ ). This follows immediately from Definition 2.1.

EXAMPLE 2.4.

(1) Classic frieze patterns, as introduced by Coxeter [4], are those frieze patterns with coefficients where all boundary entries are equal to 1 .

(2) All frieze patterns with coefficients of height 0 are of the form

$$
\begin{array}{lllllllll}
0 & a & c & 0 & & & & & \\
& 0 & b & a & 0 & & & & \\
& & 0 & c & b & 0 & & & \\
& & & 0 & a & c & 0 & & \\
& & & & 0 & b & a & 0 & \\
& & & & & 0 & c & b & 0
\end{array}
$$

with arbitrary nonzero numbers $a, b, c$. 
(3) We describe all frieze patterns with coefficients of height 1 over the positive integers. Take any four numbers $a, b, c, d \in \mathbb{Z}_{>0}$ and consider the infinite array of the form (repeated periodically)

$$
\begin{array}{cccccccc}
0 & a & x & d & 0 & & & \\
& 0 & b & y & a & 0 & & \\
& & 0 & c & z & b & 0 & \\
& & & 0 & d & w & c & 0
\end{array}
$$

It is easy to check from the local conditions that $x=z$ and $y=w$. Then this is a frieze pattern with coefficients if and only if $x y=a c+b d$. In other words, given $a, b, c, d \in \mathbb{Z}_{>0}$ we obtain for each divisor of $a c+b d$ such a frieze pattern with coefficients.

As an explicit example, we have the following frieze pattern with coefficients over $\mathbb{Z}_{>0}$ :

$$
\begin{array}{llllllll}
0 & 3 & 4 & 3 & 0 & & & \\
& 0 & 7 & 9 & 3 & 0 & & \\
& & 0 & 5 & 4 & 7 & 0 & \\
& & & 0 & 3 & 9 & 5 & 0
\end{array}
$$

For general subsets $R \subseteq \mathbb{C}$, there are many frieze patterns with coefficients having entries in $R$, and many of them do not exhibit nice symmetry properties. This is already the case for classic frieze patterns; see [5]. As in the classic case, a satisfactory theory can only be expected for tame frieze patterns.

Definition 2.5. Let $\mathcal{C}$ be a frieze pattern with coefficients as in Definition 2.1. Then $\mathcal{C}$ is called tame if every complete adjacent $3 \times 3$-submatrix of $\mathcal{C}$ has determinant 0 .

The class of tame frieze patterns with coefficients is quite large; for example, it contains all frieze patterns with coefficients without zero entries. For the sake of completeness, we include a proof of this fact here, which is well known in the classic case.

Proposition 2.6. Let $\mathcal{C}$ be a frieze pattern with coefficients as in Definition 2.1. If $c_{i, j} \neq 0$ for all $i \in \mathbb{Z}$ and $i+1 \leqslant j \leqslant n+i+2$, then $\mathcal{C}$ is tame. 
Proof. We consider an arbitrary complete adjacent $3 \times 3$-submatrix of $\mathcal{C}$, say

$$
C_{i, j}:=\left(\begin{array}{ccc}
c_{i-1, j-1} & c_{i-1, j} & c_{i-1, j+1} \\
c_{i, j-1} & c_{i, j} & c_{i, j+1} \\
c_{i+1, j-1} & c_{i+1, j} & c_{i+1, j+1}
\end{array}\right)
$$

where $i \in \mathbb{Z}$ and $i+2 \leqslant j \leqslant n+i+1$. Note that by assumption, the central element $c_{i, j}$ is nonzero. By the Sylvester identity [16], we get

$$
\operatorname{det} C_{i, j}=\frac{1}{c_{i, j}} \cdot \operatorname{det}\left(\begin{array}{ll}
c_{i-1, j-1} c_{i, j}-c_{i-1, j} c_{i, j-1} & c_{i-1, j} c_{i, j+1}-c_{i-1, j+1} c_{i, j} \\
c_{i, j-1} c_{i+1, j}-c_{i, j} c_{i+1, j-1} & c_{i, j} c_{i+1, j+1}-c_{i, j+1} c_{i+1, j}
\end{array}\right) .
$$

Using Equations $\left(E_{i-1, j-1}\right),\left(E_{i-1, j}\right),\left(E_{i, j-1}\right)$ and $\left(E_{i, j}\right)$ we deduce

$$
\operatorname{det} C_{i, j}=\frac{1}{c_{i, j}} \cdot \operatorname{det}\left(\begin{array}{cc}
c_{i, n+i+2} c_{j-1, j} & c_{i, n+i+2} c_{j, j+1} \\
c_{i+1, n+i+3} c_{j-1, j} & c_{i+1, n+i+3} c_{j, j+1}
\end{array}\right)=0,
$$

proving the claim.

We aim at showing that every tame frieze pattern with coefficients satisfies a glide symmetry. We have already seen in Remark 2.2 that the boundary entries have a glide symmetry, namely we have $c_{i, i+1}=c_{i+1, n+i+3}$ for all $i \in \mathbb{Z}$.

For extending this to all entries of the frieze patterns with coefficients, we need formulae describing how entries are 'propagated' along rows and columns, that is, how to obtain two consecutive entries in a row or column from the previous two entries. To this end, it is useful to slightly extend the definition of a frieze pattern with coefficients by introducing two extra diagonals.

REMARK 2.7. Let $\mathcal{C}$ be a frieze pattern with coefficients over $R \subseteq \mathbb{C}$ as in Definition 2.1. The corresponding extended frieze pattern with coefficients $\hat{\mathcal{C}}$ is the infinite array of the form

$$
\begin{array}{cccccccccccc}
-c_{i-2, i-1} & 0 & c_{i-1, i} & c_{i-1, i+1} & \cdots & \cdots & c_{i-1, n+i} & c_{i-1, n+i+1} & 0 & -c_{i-1, i} & \\
& -c_{i-1, i} & 0 & c_{i, i+1} & c_{i, i+2} & \cdots & \cdots & c_{i, n+i+1} & c_{i, n+i+2} & 0 & -c_{i, i+1} & \\
& & -c_{i, i+1} & 0 & c_{i+1, i+2} & c_{i+1, i+3} & \cdots & \cdots & c_{i+1, n+i+2} & c_{i+1, n+i+3} & 0 & -c_{i+1, i+2}
\end{array}
$$

That is, in addition to Definition 2.1 we set

$$
c_{i, i-1}:=-c_{i-1, i} \quad \text { and } \quad c_{i, n+i+4}:=-c_{i, i+1} \quad \text { for all } i \in \mathbb{Z} .
$$

We mention some fundamental properties of these extended frieze patterns with coefficients. 
(1) All local conditions $\left(E_{i, j}\right)$ also hold in $\hat{\mathcal{C}}$. In fact, the only new equations to check are $\left(E_{i, i}\right)$ and $\left(E_{i, n+i+3}\right)$ for $i \in \mathbb{Z}$. The former one reads as

$$
c_{i, i} c_{i+1, i+1}-c_{i, i+1} c_{i+1, i}=c_{i+1, n+i+3} c_{i, i+1},
$$

which is true since $c_{i, i}=0$ by Definition 2.1, $c_{i+1, i}=-c_{i, i+1}$ by the above definition and $c_{i+1, n+i+3}=c_{i, i+1}$ by (1). Similarly, one checks that $\left(E_{i, n+i+3}\right)$ holds.

(2) $\mathcal{C}$ is tame if and only if $\hat{\mathcal{C}}$ is tame (see Definition 2.5). In fact, the new complete adjacent $3 \times 3$-submatrices in $\hat{\mathcal{C}}$ are of the form

$$
\left(\begin{array}{ccc}
c_{i-1, i} & c_{i-1, i+1} & c_{i-1, i+2} \\
0 & c_{i, i+1} & c_{i, i+2} \\
-c_{i, i+1} & 0 & c_{i+1, i+2}
\end{array}\right) \quad \text { and } \quad\left(\begin{array}{ccc}
c_{i-1, n+i+1} & 0 & -c_{i-1, i} \\
c_{i, n+i+1} & c_{i, n+i+2} & 0 \\
c_{i+1, n+i+1} & c_{i+1, n+i+2} & c_{i+1, n+i+3}
\end{array}\right)
$$

for $i \in \mathbb{Z}$. The matrix on the left can be shown to have determinant 0 by expanding along the first column, using equation $\left(E_{i-1, i+1}\right)$ and the glide symmetry formula (1). Similarly, the matrix on the right has determinant 0 .

Instead of only adding two further diagonals to a frieze pattern with coefficients $\mathcal{C}$, one could extend $\mathcal{C}$ to an entire $S L_{2}$-tiling of the plane, still satisfying all local conditions and tameness. This is well known for classic frieze patterns and can easily be transferred to frieze patterns with coefficients. We do not introduce this viewpoint here since it is not used in the present paper.

We can now start to develop the propagation formulae. It will turn out that the following matrices are crucial for this.

Definition 2.8 ( $\mu$-matrices and $\eta$-matrices). For $c, d \in \mathbb{C}$ and $e \in \mathbb{C} \backslash\{0\}$, let

$$
\begin{aligned}
\mu(c, d, e) & :=\left(\begin{array}{cc}
0 & -\frac{d}{e} \\
1 & \frac{c}{e}
\end{array}\right), \\
\eta(c, d, e) & :=\left(\begin{array}{cc}
c & -\frac{d}{e} \\
1 & 0
\end{array}\right) .
\end{aligned}
$$

REMARK 2.9. For classic frieze patterns (that is, all coefficients are 1), the matrices $\eta(c, 1,1)$ are used throughout the literature. For our purposes, the matrices $\mu(c, d, e)$ are more convenient. They are closely linked, namely

$$
\mu(c, d, e)=\tau \eta(c, d, e)^{T} \tau,
$$


if $\tau:=\left(\begin{array}{ll}0 & 1 \\ 1 & 0\end{array}\right)$. Moreover, if $d \neq 0$, then

$$
\eta(c, d, e)^{-1}=\tau \eta(c, e, d) \tau
$$

The following result provides the propagation formulae. Note that we write the entries as they appear in the frieze pattern with coefficients in Definition 2.1, that is, as row vectors for the propagation along rows and as column vectors for the propagation along columns.

Proposition 2.10. Let $R \subseteq \mathbb{C}$ be a subset. Let $\mathcal{C}=\left(c_{i, j}\right)$ be a tame frieze pattern with coefficients over $R$ of height $n$, and $\hat{\mathcal{C}}$ the corresponding extended frieze pattern (see Remark 2.7). We write $d_{i}:=c_{i, i+1}, c_{i}:=c_{i, i+2}$ for $i \in \mathbb{Z}$ and hence $c_{i, i-1}=-c_{i-1, i}=-d_{i-1}$. Then

(1) $\left(c_{i, j-1}, c_{i, j}\right) \mu\left(c_{j-1}, d_{j}, d_{j-1}\right)=\left(c_{i, j}, c_{i, j+1}\right)$ for all $i \in \mathbb{Z}$ and $i \leqslant j \leqslant$ $n+i+3$,

(2) $\mu\left(c_{i-1}, d_{i}, d_{i-1}\right)^{T}\left(\begin{array}{c}c_{i-1, k} \\ c_{i, k}\end{array}\right)=\left(\begin{array}{c}c_{i, k} \\ c_{i+1, k}\end{array}\right)$ for all $k \in \mathbb{Z}$ and $k-n-3 \leqslant i \leqslant k$.

Proof. We prove the first part; the proof of the second part is similar.

We first consider the cases $j=i$ and $j=i+1$ separately. For $j=i$, we have

$$
\left(c_{i, i-1}, c_{i, i}\right) \mu\left(c_{i-1}, d_{i}, d_{i-1}\right)=\left(-d_{i-1}, 0\right)\left(\begin{array}{cc}
0 & -\frac{d_{i}}{d_{i-1}} \\
1 & \frac{c_{i-1}}{d_{i-1}}
\end{array}\right)=\left(0, d_{i}\right)=\left(c_{i, i}, c_{i, i+1}\right)
$$

as claimed. For $j=i+1$, we similarly get

$$
\left(c_{i, i}, c_{i, i+1}\right) \mu\left(c_{i}, d_{i+1}, d_{i}\right)=\left(0, d_{i}\right)\left(\begin{array}{cc}
0 & -\frac{d_{i+1}}{d_{i}} \\
1 & \frac{c_{i}}{d_{i}}
\end{array}\right)=\left(d_{i}, c_{i}\right)=\left(c_{i, i+1}, c_{i, i+2}\right) .
$$

Now suppose $i+2 \leqslant j \leqslant n+i+3$. Then we consider the following complete adjacent $3 \times 3$-submatrix of the extended frieze pattern

$$
M=\left(\begin{array}{ccc}
c_{i, j-1} & c_{i, j} & c_{i, j+1} \\
c_{i+1, j-1} & c_{i+1, j} & c_{i+1, j+1} \\
c_{i+2, j-1} & c_{i+2, j} & c_{i+2, j+1}
\end{array}\right)
$$


The first two columns of $M$ cannot be linearly dependent because the upper left $2 \times 2$-submatrix has determinant $d_{i} d_{j-1} \neq 0$ by Definition 2.1 and Remark 2.2.

But $\mathcal{C}$ is tame by assumption. Hence the determinant of $M$ is zero; so there are suitable numbers $s, t$ such that

$$
M=\left(\begin{array}{ccc}
c_{i, j-1} & c_{i, j} & s c_{i, j-1}+t c_{i, j} \\
c_{i+1, j-1} & c_{i+1, j} & s c_{i+1, j-1}+t c_{i+1, j} \\
c_{i+2, j-1} & c_{i+2, j} & s c_{i+2, j-1}+t c_{i+2, j}
\end{array}\right)
$$

Now Equations $\left(E_{i, j}\right)$ and $\left(E_{i, j-1}\right)$ imply

$$
\begin{aligned}
d_{i} d_{j} & =c_{i, i+1} c_{j, j+1}=c_{i+1, n+i+3} c_{j, j+1} \\
& =c_{i, j} c_{i+1, j+1}-c_{i, j+1} c_{i+1, j} \\
& =c_{i, j}\left(s c_{i+1, j-1}+t c_{i+1, j}\right)-c_{i+1, j}\left(s c_{i, j-1}+t c_{i, j}\right) \\
& =s\left(c_{i, j} c_{i+1, j-1}-c_{i+1, j} c_{i, j-1}\right) \\
& =-s c_{i+1, n+i+3} c_{j-1, j}=-s d_{i} d_{j-1},
\end{aligned}
$$

and we conclude that $s=-d_{j} / d_{j-1}$. Thus we see from the shape of the matrix $M$ that for fixed $j-1$, there is a $t_{j-1}=t$ such that for all $i$ we have

$$
\left(c_{i, j-1}, c_{i, j}\right)\left(\begin{array}{cc}
0 & -\frac{d_{j}}{d_{j-1}} \\
1 & t_{j-1}
\end{array}\right)=\left(c_{i, j}, c_{i, j+1}\right) .
$$

In particular, this equation holds for $i=j-1$ and we get

$$
c_{j-1}=c_{j-1, j+1}=-\underbrace{c_{j-1, j-1}}_{=0} \frac{d_{j}}{d_{j-1}}+c_{j-1, j} t_{j-1}=c_{j-1, j} t_{j-1}=d_{j-1} t_{j-1},
$$

hence $t_{j-1}=c_{j-1} / d_{j-1}$. Altogether we obtain

$$
\left(c_{i, j-1}, c_{i, j}\right) \mu\left(c_{j-1}, d_{j}, d_{j-1}\right)=\left(c_{i, j-1}, c_{i, j}\right)\left(\begin{array}{cc}
0 & -\frac{d_{j}}{d_{j-1}} \\
1 & \frac{c_{j-1}}{d_{j-1}}
\end{array}\right)=\left(c_{i, j}, c_{i, j+1}\right),
$$

as claimed.

As a consequence, we can give a useful formula for determining the entries of the frieze pattern with coefficients from the corresponding $\mu$-matrices. 
COROLlaRY 2.11. Let $R \subseteq \mathbb{C}$ be a subset. Let $\mathcal{C}=\left(c_{i, j}\right)$ be a tame frieze pattern with coefficients over $R$ of height $n$, and as before set $d_{i}=c_{i, i+1}, c_{i}=c_{i, i+2}$ for $i \in \mathbb{Z}$. Then we have:

(1) $\prod_{k=i}^{j} \mu\left(c_{k-1}, d_{k}, d_{k-1}\right)=\left(1 / d_{i-1}\right)\left(\begin{array}{cc}-c_{i, j} & -c_{i, j+1} \\ c_{i-1, j} & c_{i-1, j+1}\end{array}\right)$ for all $i \in \mathbb{Z}$ and $i-1 \leqslant j \leqslant n+i+2$;

(2) $\prod_{k=1}^{n+3} \mu\left(c_{k-1}, d_{k}, d_{k-1}\right)=-\mathrm{id}$.

Proof. We consider the extended frieze pattern with coefficients as in Remark 2.7; in particular, we set $c_{i, i-1}=-d_{i-1}$ for all $i \in \mathbb{Z}$.

(1) From the first part of Proposition 2.10, we know that

$$
\begin{aligned}
& \left(\begin{array}{cc}
-d_{i-1} & 0 \\
0 & d_{i-1}
\end{array}\right) \cdot\left(\prod_{k=i}^{j} \mu\left(c_{k-1}, d_{k}, d_{k-1}\right)\right) \\
& =\left(\begin{array}{cc}
c_{i, i-1} & c_{i, i} \\
c_{i-1, i-1} & c_{i-1, i}
\end{array}\right) \cdot\left(\prod_{k=i}^{j} \mu\left(c_{k-1}, d_{k}, d_{k-1}\right)\right) \\
& =\left(\begin{array}{cc}
c_{i, j} & c_{i, j+1} \\
c_{i-1, j} & c_{i-1, j+1}
\end{array}\right)
\end{aligned}
$$

and the claim follows.

(2) By the first part for $i=1$ and $j=n+3$, we have

$$
\prod_{k=1}^{n+3} \mu\left(c_{k-1}, d_{k}, d_{k-1}\right)=\frac{1}{d_{0}}\left(\begin{array}{cc}
-c_{1, n+3} & -c_{1, n+4} \\
c_{0, n+3} & c_{0, n+4}
\end{array}\right) \text {. }
$$

Now the claim follows since $c_{0, n+3}=0=c_{1, n+4}$ by Definition 2.1, $c_{0, n+4}=$ $-c_{0,1}=-d_{0}$ by Remark 2.7 and $c_{1, n+3}=c_{0,1}=d_{0}$ by Equation (1).

As another main result of this section, we can now prove that the entries of a tame frieze pattern with coefficients are invariant under a glide reflection. This will become crucial in the following sections.

THEOREM 2.12. Let $R \subseteq \mathbb{C}$ be a subset. Let $\mathcal{C}=\left(c_{i, j}\right)$ be a tame frieze pattern with coefficients over $R$ of height $n$. Then for all entries of $\mathcal{C}$, we have

$$
c_{i, j}=c_{j, n+i+3} .
$$

Proof. We consider the $i$ th row and the $(n+i+3)$ th column in the extended frieze pattern corresponding to $\mathcal{C}$; see Figure 2 . We propagate along the $(n+i+3)$ th 


$$
\begin{aligned}
& \begin{array}{llllllllll}
-d_{i-1} & 0 & d_{i} & \ldots & c_{i, j} & \ldots & c_{i, n+i+2} & 0 & -d_{i}
\end{array} \\
& c_{i+1, n+i+3} \quad 0 \\
& c_{i+2, n+i+3} \\
& \vdots \\
& c_{j-1, n+i+3} \\
& c_{j, n+i+3} \\
& c_{j+1, n+i+3} \\
& d_{n+i+2} \\
& 0 \\
& -d_{n+i+3}
\end{aligned}
$$

Figure 2. The $i$ th row and the $(n+i+3)$ th column in the extended frieze pattern.

column using Proposition 2.10(2) and get

$$
\begin{aligned}
\left(\begin{array}{c}
c_{j, n+i+3} \\
c_{j+1, n+i+3}
\end{array}\right) & =\mu\left(c_{j-1}, d_{j}, d_{j-1}\right)^{T} \ldots \mu\left(c_{i}, d_{i+1}, d_{i}\right)^{T} \mu\left(c_{i-1}, d_{i}, d_{i-1}\right)^{T}\left(\begin{array}{c}
-d_{i-1} \\
0
\end{array}\right) \\
& =\left(\prod_{k=i}^{j} \mu\left(c_{k-1}, d_{k}, d_{k-1}\right)\right)^{T}\left(\begin{array}{c}
-d_{i-1} \\
0
\end{array}\right) .
\end{aligned}
$$

This implies that

$$
\begin{aligned}
c_{j, n+i+3} & =-d_{i-1}\left(\left(\prod_{k=i}^{j} \mu\left(c_{k-1}, d_{k}, d_{k-1}\right)\right)^{T}\right)_{1,1} \\
& =-d_{i-1}\left(\prod_{k=i}^{j} \mu\left(c_{k-1}, d_{k}, d_{k-1}\right)\right)_{1,1}=c_{i, j},
\end{aligned}
$$

where the last equality holds by Corollary 2.11 .

REMARK 2.13. It follows from Theorem 2.12 that $c_{i, j}=c_{n+i+3, n+j+3}$ for all entries of $\mathcal{C}$, that is, the diagonals of $\mathcal{C}$ are periodic of period $n+3$. Note that this is exactly what is known as Zamolodchikov periodicity in type $A$; see [9]. 


$\begin{array}{cccccccc} & \ddots & & & & & \ddots & \\ 0 & c_{1,2} & c_{1,3} & \ldots & \ldots & \ldots & c_{1, n+3} & 0 \\ & 0 & c_{2,3} & c_{2,4} & \ldots & \ldots & c_{2, n+3} & \ddots \\ & \ddots & \ddots & \ddots & & \vdots & \\ & & \ddots & \ddots & \ddots & \vdots & \\ & & & 0 & c_{n+1, n+2} & c_{n+1, n+3} & \\ & & & & 0 & c_{n+2, n+3} & \ddots \\ & & & & & 0 & \ddots \\ & & & & & & 0\end{array}$

Figure 3. Fundamental domain for the glide symmetry of a frieze pattern with coefficients.

\section{Ptolemy relations}

In the previous section, we have seen that every tame frieze pattern with coefficients over some subset $R \subseteq \mathbb{C}$ satisfies a glide symmetry; see Theorem 2.12. More precisely, Theorem 2.12 states that the triangular part of the frieze pattern with coefficients given in Figure 3 forms a fundamental domain for the action of the glide symmetry. Note that the indices of the entries in this fundamental domain are in bijection with the edges and diagonals of a regular $(n+3)$-gon (viewed as pairs of vertices), with vertices labelled $1,2, \ldots, n+3$. This means that we can view every tame frieze pattern with coefficients of height $n$ over $R$ as a map on the edges and diagonals of a regular $(n+3)$-gon with values in $R$.

CONVEntion. We use the notion (tame) frieze pattern with coefficients for an infinite array as in Definition 2.1 and the notion (tame) frieze with coefficients for a corresponding map from edges and diagonals of a regular polygon.

The entries in a frieze (pattern) with coefficients are tightly connected by many remarkable equations, in addition to the local conditions given in Equations $\left(E_{i, j}\right)$ in Definition 2.1.

Definition 3.1. Let $\mathcal{C}=\left(c_{i, j}\right)$ be a tame frieze with coefficients over $R \subseteq \mathbb{C}$ on a regular $m$-gon. We say that $\mathcal{C}$ satisfies the Ptolemy relation for the indices $1 \leqslant i \leqslant j \leqslant k \leqslant \ell \leqslant m$ if the following equation holds:

$$
c_{i, k} c_{j, \ell}=c_{i, \ell} c_{j, k}+c_{i, j} c_{k, \ell} . \quad\left(E_{i, j, k, \ell}\right)
$$




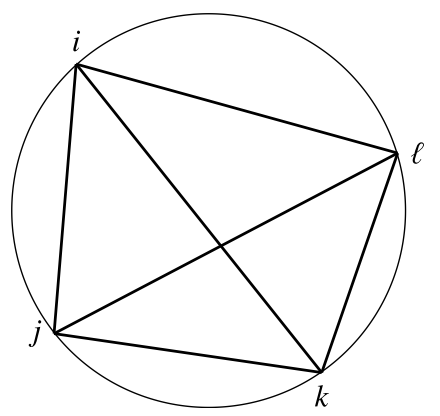

Figure 4. The Ptolemy relation $\left(E_{i, j, k, \ell}\right)$.

\section{REMARK 3.2.}

(1) By Definition 2.1, the entries $c_{i, i}$ are zero for all $i \in \mathbb{Z}$. This implies that the Equations $\left(E_{i, j, k, \ell}\right)$ always hold if there are equalities among the numbers $i, j, k, \ell$.

(2) If $i<j<k<\ell$, then the Ptolemy relation $\left(E_{i, j, k, \ell}\right)$ can be visualized as in Figure 4. The Ptolemy relation asks that in the quadrilateral, the product of the labels on the diagonals equals the sum of the products of labels of opposite sides; see Ptolemy's theorem from elementary Euclidean geometry.

(3) The local conditions for a frieze pattern from Definition 2.1 are a special case of Ptolemy relations. Namely, the local condition $\left(E_{i, j}\right)$ is equal to the Ptolemy relation $\left(E_{i, i+1, j, j+1}\right)$ (using $c_{i, i+1}=c_{i+1, n+i+3}$ by Remark 2.2).

The next result now extends a fundamental property for classic friezes (see [4, Equation (5.7)]) to friezes with coefficients.

THEOREM 3.3. Every tame frieze with coefficients over some subset $R \subseteq \mathbb{C}$ satisfies all Ptolemy relations.

Proof. Let $\mathcal{C}$ be a tame frieze on a regular $m$-gon, where $m \geqslant 3$. Take any four vertices $1 \leqslant i \leqslant j \leqslant k \leqslant \ell \leqslant m$ of the regular $m$-gon. By Corollary 2.11, we have that

$$
M_{i+1, j}:=\prod_{k=i+1}^{j} \mu\left(c_{k-1}, d_{k}, d_{k-1}\right)=\frac{1}{d_{i}}\left(\begin{array}{cc}
-c_{i+1, j} & -c_{i+1, j+1} \\
c_{i, j} & c_{i, j+1}
\end{array}\right) .
$$


Using $M_{i+1, k}=M_{i+1, j} M_{j+1, k}, \quad M_{j+1, \ell}=M_{j+1, k} M_{k+1, \ell}$ and $M_{i+1, \ell}=$ $M_{i+1, j} M_{j+1, k} M_{k+1, \ell}$, we get from the $(2,1)$-entries of the matrices that

$$
\begin{aligned}
\frac{1}{d_{i}} c_{i, k}= & \frac{1}{d_{i} d_{j}}\left(c_{i, j+1} c_{j, k}-c_{j+1, k} c_{i, j}\right), \\
\frac{1}{d_{j}} c_{j, \ell}= & \frac{1}{d_{j} d_{k}}\left(c_{j, k+1} c_{k, l}-c_{k+1, \ell} c_{j, k}\right), \\
\frac{1}{d_{i}} c_{i, \ell}= & \frac{1}{d_{i} d_{j} d_{k}}\left(c_{i, j+1} c_{j, k+1} c_{k, \ell}-c_{i, j+1} c_{k+1, \ell} c_{j, k}\right. \\
& \left.+c_{j+1, k} c_{k+1, \ell} c_{i, j}-c_{j+1, k+1} c_{i, j} c_{k, \ell}\right) .
\end{aligned}
$$

With these three equations and $\left(E_{j, k}\right)$, we conclude:

$$
\begin{aligned}
& \frac{1}{d_{i} d_{j}}\left(c_{i, j} c_{k, \ell}+c_{j, k} c_{i, \ell}-c_{i, k} c_{j, \ell}\right) \\
& \stackrel{(5),(6)}{=} \frac{1}{d_{i} d_{j}}\left(c_{i, j} c_{k, \ell}+c_{j, k} c_{i, \ell}\right) \\
& \quad-\frac{1}{d_{i} d_{j}^{2} d_{k}}\left(c_{i, j+1} c_{j, k}-c_{j+1, k} c_{i, j}\right)\left(c_{j, k+1} c_{k, l}-c_{k+1, \ell} c_{j, k}\right) \\
& \stackrel{(7)}{=} \frac{1}{d_{i} d_{j}}\left(c_{i, j} c_{k, \ell}+c_{j, k} c_{i, \ell}\right) \\
& \quad-\frac{1}{d_{i} d_{j}^{2} d_{k}}\left(c_{i, j+1} c_{j, k}-c_{j+1, k} c_{i, j}\right)\left(c_{j, k+1} c_{k, l}-c_{k+1, \ell} c_{j, k}\right) \\
& \quad+\frac{1}{d_{j}} c_{j, k}\left(\frac { 1 } { d _ { i } d _ { j } d _ { k } } \left(c_{i, j+1} c_{j, k+1} c_{k, \ell}-c_{i, j+1} c_{k+1, \ell} c_{j, k}\right.\right. \\
& \left.\left.\quad+c_{j+1, k} c_{k+1, \ell} c_{i, j}-c_{j+1, k+1} c_{i, j} c_{k, \ell}\right)-\frac{1}{d_{i}} c_{i, \ell}\right) \\
& =\frac{1}{d_{i} d_{j}} c_{i, j} c_{k, \ell}\left(1-\frac{1}{d_{j} d_{k}}\left(c_{j, k} c_{j+1, k+1}-c_{j, k+1} c_{j+1, k}\right)\right) \stackrel{\left(E_{j, k}\right)}{=} 0 .
\end{aligned}
$$

Hence we obtain $c_{i, j} c_{k, \ell}+c_{j, k} c_{i, \ell}=c_{i, k} c_{j, \ell}$, that is, the Ptolemy relation $\left(E_{i, j, k, \ell}\right)$ holds.

REMARK 3.4. We have now seen that a tame frieze pattern with coefficients as in Definition 2.1 is basically the same as a map on edges and diagonals of a polygon satisfying all Ptolemy relations. This means that indeed the local condition of a 
frieze pattern with coefficients produces the cluster variables of a Ptolemy cluster algebra with coefficients; see for instance [14, Section 1] for a description of the Ptolemy cluster algebra (also known as cluster algebra of Dynkin type $A$ with nontrivial coefficients).

\section{Frieze patterns from subpolygons}

From now on, we consider frieze patterns with coefficients over positive integers.

Using the cluster algebra viewpoint as in Remark 3.4, one can see that for every boundary sequence of positive integers (of length $n+3$ ) there exists a frieze pattern with coefficients over the positive integers. Indeed, a theorem on cluster algebras, the Laurent phenomenon, implies that every cluster variable can be expressed as a rational function whose denominator is a monomial in the initial nonfrozen cluster variables (that is, the coefficients do not appear in the denominator) and whose numerator is a positive sum (no minus signs appear) of products of frozen and nonfrozen initial cluster variables. Hence, specializing the initial cluster variables to 1 gives a frieze pattern of positive integers with the given boundary sequence.

EXAMPLE 4.1. Consider a boundary sequence $(a, b, c, d, e)$, that is, a sequence of frozen variables. The corresponding frieze pattern of height 2 has the form

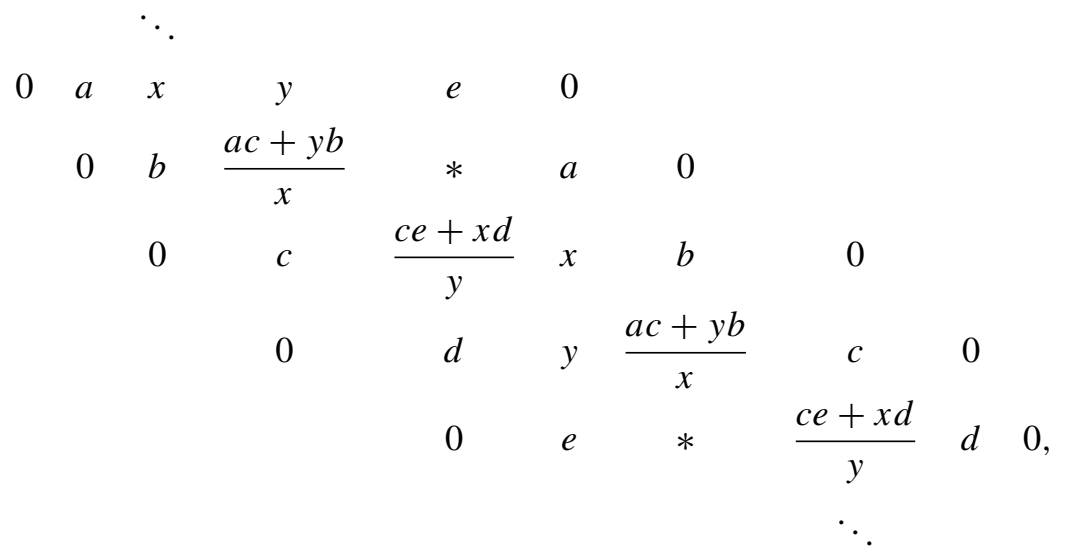

where $x, y$ denote the cluster variables in the chosen initial cluster and $*=($ bey + ace $+a x d) / x y$. Specializing the initial frozen cluster variables to any positive integers and the nonfrozen cluster variables $x, y$ to 1 yields a frieze 
pattern of positive integers. As an explicit example, we have a frieze pattern

$\begin{array}{cccccccccc}0 & 3 & 1 & 1 & 7 & 0 & & & & \\ & 0 & 2 & 302 & 2126 & 3 & 0 & & & \\ & & 0 & 100 & 704 & 1 & 2 & 0 & & \\ & & 0 & 4 & 1 & 302 & 100 & 0 & \\ & & & 0 & 7 & 2126 & 704 & 4 & 0 \\ & & & & & & \ddots & & \end{array}$

In the previous section, we have seen that a tame frieze pattern with coefficients of height $n$ can be seen as a map from edges and diagonals of an $(n+3)$-gon such that all Ptolemy relations are satisfied.

For classic friezes over $\mathbb{N}$, there is a beautiful combinatorial description via triangulations of polygons [3].

Convention. From now on, we use the notion classic Conway-Coxeter frieze (pattern) for a classic frieze (pattern) over the positive integers $\mathbb{N}$.

Our aim is to connect the more general theory of friezes with coefficients over $\mathbb{N}$ with the theory of classic Conway-Coxeter friezes.

From the viewpoint of polygons, there is an obvious way to obtain friezes with coefficients from classic Conway-Coxeter friezes. Namely, take any classic Conway-Coxeter frieze $\mathcal{C}$. This corresponds to a triangulation of a regular polygon [3], that is, it is given as a map $f_{\mathcal{C}}$ from edges and diagonals of the polygon to $\mathbb{N}$, where $f_{\mathcal{C}}$ takes value 1 on the edges and the diagonals forming the triangulation.

Now cut out any subpolygon and restrict the map $f_{\mathcal{C}}$ to this subpolygon. Clearly, the restricted map still satisfies all Ptolemy relations of the subpolygon, that is, the restriction yields a frieze with coefficients.

EXAMPLE 4.2. We consider the triangulation of a hexagon on the left in Figure 5 and the corresponding Conway-Coxeter frieze. For instance, we can cut out the subpolygon highlighted by thick lines on the right in Figure 5. This yields a frieze with coefficients with boundary sequence $(1,1,2,2)$, and diagonal values 1,4 as indicated in the figure.

The fundamental question then is as follows: Which friezes with coefficients over $\mathbb{N}$ can be obtained from classic Conway-Coxeter friezes by cutting out subpolygons? 

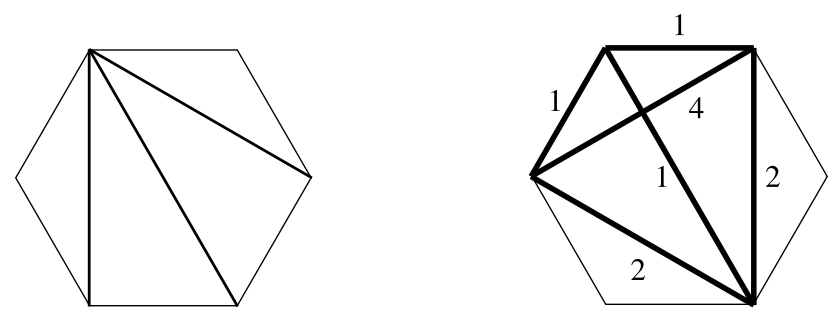

Figure 5. A frieze with coefficients cut out of a Conway-Coxeter frieze.

The following result gives some restrictions for the smallest case of triangles.

LemmA 4.3. Let $\mathcal{C}=\left(c_{i, j}\right)$ be a classic Conway-Coxeter frieze and $i \leqslant j \leqslant k$. Then the greatest common divisor of any two of the numbers $c_{i, j}, c_{j, k}, c_{i, k}$ divides the third number. In particular, $\operatorname{gcd}\left(c_{i, j}, c_{j, k}\right)=\operatorname{gcd}\left(c_{j, k}, c_{k, i}\right)=\operatorname{gcd}\left(c_{i, j}, c_{k, i}\right)$.

Proof. By symmetry and relabelling, it suffices to show that $\operatorname{gcd}\left(c_{j, k}, c_{i, k}\right)$ divides $c_{i, j}$.

If $i=j$, then the assertion holds since $c_{i, i}=0$. If $j=k$, then $\operatorname{gcd}\left(c_{j, k}, c_{i, k}\right)=$ $c_{i, k}=c_{i, j}$ and the claim follows. If $j=k-1$, then $\operatorname{gcd}\left(c_{j, k}, c_{i, k}\right)=\operatorname{gcd}\left(1, c_{i, k}\right)=1$, which clearly divides $c_{i, j}$.

So from now on, we can assume that $i<j<k-1$. Then the Ptolemy relation for the crossing diagonals $(i, k-1)$ and $(j, k)$ implies that

$$
c_{i, k-1} c_{j, k}=c_{j, k-1} c_{i, k}+c_{i, j} c_{k-1, k}=c_{j, k-1} c_{i, k}+c_{i, j} .
$$

Therefore, any common divisor of $c_{j, k}$ and $c_{i, k}$ divides $c_{i, j}$ and the claim follows.

\section{EXAMPLE 4.4.}

(1) We have seen in Example 2.4 that any frieze with coefficients of height 0 over $\mathbb{N}$ is given by three numbers $a, b, c$; these are the values attached to the edges of the corresponding triangle. Lemma 4.3 implies that such a triangle can only be cut out of a classic Conway-Coxeter frieze if the greatest common divisor of two of $a, b, c$ divides the third. For instance, a triangle with values 1,2,2 cannot come from a classic Conway-Coxeter frieze.

In the next section, we will consider triangles in more detail and will obtain a complete characterization for which triangles with triples $a, b, c$ can be cut out of a classic Conway-Coxeter frieze. 
(2) Consider the following frieze with coefficients on a square:

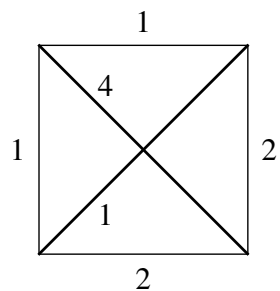

This square cannot be cut out of a classic Conway-Coxeter frieze because it contains a triangle with values $1,2,2$, which cannot come from a classic Conway-Coxeter frieze by part (1).

We have seen in Examples 4.2 and 4.4 that whether a frieze with coefficients can be cut out of a classic Conway-Coxeter frieze does not only depend on the boundary sequence.

Building on the Euclidean algorithm, the next result shows that triangles can be cut out of a classic Conway-Coxeter frieze if one of the values on the edges is 1 and the other two values are coprime. Note that the coprimeness condition has to be satisfied in this case by Lemma 4.3. This is a known property of classic Conway-Coxeter friezes (see [12, Section 2.5, Fact 3(iii)]); for the convenience of the reader, we include a proof here.

LemmA 4.5. Let $a, b \in \mathbb{Z}_{\geqslant 0}$ with $\operatorname{gcd}(a, b)=1$. Then there exists a classic Conway-Coxeter frieze $\mathcal{C}=\left(c_{i, j}\right)$ and $a k \geqslant 1$ such that $c_{1, k}=a, c_{k, k+1}=1$ and $c_{1, k+1}=b$.

Proof. Note that by assumption not both of $a, b$ can be zero, since $\operatorname{gcd}(0,0)=0$. If one of them is zero, say $a=0$, then $b=\operatorname{gcd}(0, b)=\operatorname{gcd}(a, b)=1$ and the assertion holds with $k=1$; in fact, use the unique classic Conway-Coxeter frieze on a triangle.

So from now on we can assume that $a, b \in \mathbb{N}$. We show how the Euclidean algorithm leads to a triangulation of a polygon, and hence to a classic ConwayCoxeter frieze, with the required values.

First, we perform the Euclidean algorithm, where we can assume that $a \geqslant b$. Set $r_{0}=a$ and $r_{1}=b$; then

$$
\begin{aligned}
a & =q_{1} b+r_{2} \\
b & =q_{2} r_{2}+r_{3} \\
r_{2} & =q_{3} r_{3}+r_{4}
\end{aligned}
$$




$$
\begin{aligned}
r_{\ell-2} & =q_{\ell-1} r_{\ell-1}+r_{\ell} \\
r_{\ell-1} & =q_{\ell} r_{\ell}+r_{\ell+1} \\
r_{\ell} & =q_{\ell+1} r_{\ell+1}+0 .
\end{aligned}
$$

Note that by assumption, we have $r_{\ell+1}=\operatorname{gcd}(a, b)=1$ and $q_{\ell+1}=r_{\ell}$.

We now describe how to obtain from this data a suitable triangulation of a polygon.

Consider the usual number line, and the integral points as vertices. Start with the edge connecting 1 and 2 . Then draw $q_{\ell+1}$ arcs from 2 to the left to the next available vertices, that is, to the vertices $0,-1, \ldots,-q_{\ell+1}+1$. Then from vertex $-q_{\ell+1}+1$ draw $q_{\ell}$ arcs to the right to the next available vertices. Continue in this alternating fashion by drawing $q_{\ell-1}, q_{\ell-2}, \ldots, q_{3}, q_{2}, q_{1}$ arcs to the left and right, respectively.

This gives a triangulation of a polygon whose vertices are the integers that are attached to one of the arcs described above. (The rest of the number line is now disregarded.) Note that this polygon is an $m:=\left(2+\sum_{j=1}^{\ell+1} q_{j}\right)$-gon. We keep the label for vertex 1 , but then number the vertices of this polygon as usual consecutively by $1,2, \ldots, m$.

We claim that the classic Conway-Coxeter frieze to this triangulation of the $m$-gon has the desired properties. For this, we use a well-known combinatorial algorithm for computing arbitrary frieze entries from the triangulation (see [2]) starting from the vertex 1 . The values attached to the vertices by this algorithm are then the frieze entries $c_{1, j}$.

The endpoint of the last of $q_{\ell+1}$ arcs to the left gets the label $q_{\ell+1}=r_{\ell}$. Then the endpoints of the $q_{\ell}$ arcs to the right get the labels $r_{\ell}+1,2 r_{\ell}+1, \ldots, q_{\ell} r_{\ell}+$ $1=r_{\ell-1}$. Then the last of the endpoints of the $q_{\ell-1}$ arcs to the left gets the label $q_{\ell-1} r_{\ell-1}+r_{\ell}=r_{\ell-2}$. Eventually, we get two consecutive vertices $k+1$ and $k$ in the polygon, which get assigned the values $r_{1}=b$ and $r_{0}=a$.

This means that we have constructed a classic Conway-Coxeter frieze $\mathcal{C}=$ $\left(c_{i, j}\right)$ with $c_{k, k+1}=1, c_{1, k}=a$ and $c_{1, k+1}=b$.

\section{Triangles in Conway-Coxeter friezes}

We have seen in Section 4 that cutting out any subpolygon of a classic ConwayCoxeter frieze yields a frieze with coefficients over $\mathbb{N}$. In this section, we give an explicit description of all possible values on the edges of triangles cut out of classic Conway-Coxeter friezes. Recall that we have already obtained some results on triples $(a, b, c)$ appearing as labels of a triangle cut out of a classic Conway-Coxeter frieze. Namely, the greatest common divisor of any two of the 
numbers divides the third (see Lemma 4.3), and for any pair $a, b$ of coprime natural numbers, the triple $(a, 1, b)$ appears as a triangle in some classic ConwayCoxeter frieze (see Lemma 4.5).

However, the complete classification of possible triples is rather subtle and will be the main topic of this section; see Theorem 5.11 below for a precise statement of our main result.

The following auxiliary result shows that given three vertices of an $n$-gon, any triangulation of the $n$-gon contains a triangle, which separates the given points.

LEMMA 5.1. Let $\mathcal{C}=\left(c_{i, j}\right)$ be a classic Conway-Coxeter frieze on an $n$-gon. If $1 \leqslant i<j<k \leqslant n$, then there exist $i^{\prime}, j^{\prime}, k^{\prime}$ with $i \leqslant i^{\prime} \leqslant j \leqslant j^{\prime} \leqslant k \leqslant k^{\prime}$ or $k^{\prime} \leqslant i \leqslant i^{\prime} \leqslant j \leqslant j^{\prime} \leqslant k$ such that

$$
c_{i^{\prime}, j^{\prime}}=c_{j^{\prime}, k^{\prime}}=c_{k^{\prime}, i^{\prime}}=1 .
$$

Proof. Let $\mathcal{T}$ be the triangulation of an $n$-gon corresponding to the classic Conway-Coxeter frieze $\mathcal{C}$, that is, $\mathcal{T}$ is the set of edges and diagonals of the $n$-gon mapped to 1 under the map $f_{\mathcal{C}}$ mentioned in Section 4. Any diagonal of $\mathcal{T}$ divides the $n$-gon into two subpolygons.

If each diagonal of $\mathcal{T}$ has the property that the three vertices $i, j, k$ are contained in one of the two subpolygons, then the triangle given by $i, j, k$ is a triangle of the triangulation $\mathcal{T}$ and we are done by choosing $i=i^{\prime}, j=j^{\prime}$ and $k=k^{\prime}$.

So we can choose a diagonal $(u, v)$ of $\mathcal{T}$, which separates the three vertices. By symmetry and possibly relabelling the vertices, we can assume that we have the situation as given in Figure 6. Moreover, we choose the diagonal $(u, v) \in \mathcal{T}$ such that $|i-u|$ and $|v-j|$ are minimal. That is, of all the diagonals of $\mathcal{T}$ separating the three vertices in this way, we choose the one closest to the diagonal $(i, j)$. Note that the latter need not be in $\mathcal{T}$, but the cases $i=u$ and $j=v$ are allowed.

If $j \neq i+1$, then the diagonal $(u, v)$ of $\mathcal{T}$ is part of a triangle in the left subpolygon. By minimality of $|i-u|$ and $|j-v|$, the third endpoint $w$ of this triangle must satisfy $i+1 \leqslant w \leqslant j-1$. Now set $i^{\prime}=w, j^{\prime}=v, k^{\prime}=u$, and we are done.

This leaves us with the case that $j=i+1$. But then the edge $(i, i+1)$ is part of a triangle of $\mathcal{T}$, and it is not hard to check that the assertion of the lemma holds in this case.

We now come to a crucial reformulation of the problem of describing the triangles, which appear as subpolygons in classic Conway-Coxeter friezes. Namely, we are going to show that such triangles correspond (but not bijectively!) to certain tuples of coprime pairs of numbers. 


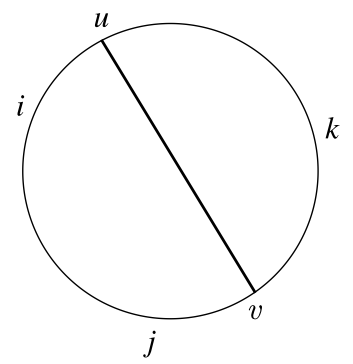

Figure 6. A diagonal separating three vertices.

To understand the following explanations, Figure 7 might be helpful. In particular, the Ptolemy relations required in the proof of Proposition 5.3 are easy to read from the picture.

\section{DEFINITION 5.2. Let}

$$
S:=\left\{\left(a_{1}, a_{2}, b_{1}, b_{2}, c_{1}, c_{2}\right) \in \mathbb{Z}^{6} \mid \operatorname{gcd}\left(a_{1}, a_{2}\right)=\operatorname{gcd}\left(b_{1}, b_{2}\right)=\operatorname{gcd}\left(c_{1}, c_{2}\right)=1\right\}
$$

and $S_{\geqslant 0}:=S \cap \mathbb{Z}_{\geqslant 0}^{6}$. Moreover, let $\Delta: S \rightarrow \mathbb{Z}^{3}$ be the map

$$
\begin{aligned}
& \Delta\left(\left(a_{1}, a_{2}, b_{1}, b_{2}, c_{1}, c_{2}\right)\right) \\
& \quad:=\left(b_{1} c_{1}+b_{1} c_{2}+b_{2} c_{2}, a_{1} c_{1}+a_{2} c_{1}+a_{2} c_{2}, a_{1} b_{1}+a_{1} b_{2}+a_{2} b_{2}\right) .
\end{aligned}
$$

The following result explains the relevance of the sets and maps in Definition 5.2 for our purposes; namely, every triple of labels of a triangle cut out of a classic Conway-Coxeter frieze is in the image of the map $\Delta$.

Proposition 5.3. Let $\mathcal{C}=\left(c_{i, j}\right)$ be a classic Conway-Coxeter frieze. If $i<j<k$, then there exists a tuple $\left(a_{1}, a_{2}, b_{1}, b_{2}, c_{1}, c_{2}\right) \in S_{\geqslant 0}$ such that $\left(c_{i, j}, c_{j, k}, c_{k, i}\right)=\Delta\left(\left(a_{1}, a_{2}, b_{1}, b_{2}, c_{1}, c_{2}\right)\right)$.

Proof. Choose $i^{\prime}, j^{\prime}, k^{\prime}$ as in Lemma 5.1, that is,

$$
c_{i^{\prime}, j^{\prime}}=c_{j^{\prime}, k^{\prime}}=c_{k^{\prime}, i^{\prime}}=1 \text {. }
$$

By Lemma 4.3,

$$
\operatorname{gcd}\left(c_{k, k^{\prime}}, c_{j^{\prime}, k}\right)=\operatorname{gcd}\left(c_{i, i^{\prime}}, c_{k^{\prime}, i}\right)=\operatorname{gcd}\left(c_{j, j^{\prime}}, c_{i^{\prime}, j}\right)=1 .
$$

Using the Ptolemy relations (Theorem 3.3 and also Remark 3.2(1)) and Equation (8), we obtain

$$
\begin{aligned}
c_{i^{\prime}, k} & =c_{k, k^{\prime}}+c_{j^{\prime}, k}, \\
c_{j^{\prime}, i} & =c_{i, i^{\prime}}+c_{k^{\prime}, i}, \\
c_{k^{\prime}, j} & =c_{j, j^{\prime}}+c_{i^{\prime}, j} .
\end{aligned}
$$




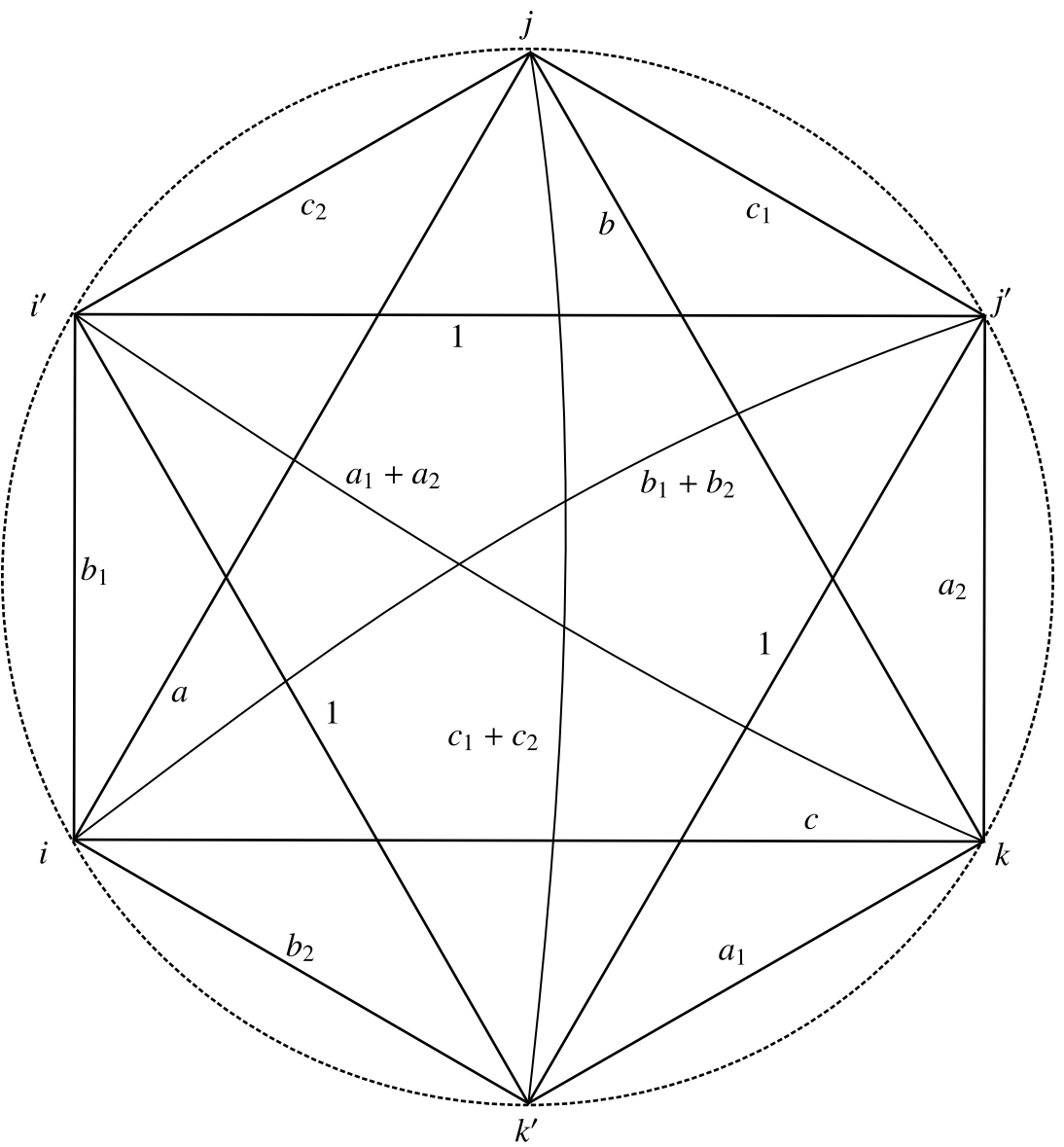

Figure 7. A triangle $(a, b, c)$ and its surroundings.

Again by the Ptolemy relations, we now obtain

$$
\begin{aligned}
& c_{i, j}=c_{i, i^{\prime}} c_{k^{\prime}, j}+c_{k^{\prime}, i} c_{i^{\prime}, j}=c_{i, i^{\prime}} c_{j, j^{\prime}}+c_{i, i^{\prime}} c_{i^{\prime}, j}+c_{k^{\prime}, i} c_{i^{\prime}, j}, \\
& c_{j, k}=c_{i^{\prime}, k} c_{j, j^{\prime}}+c_{j^{\prime}, k} c_{i^{\prime}, j}=c_{k, k^{\prime}} c_{j, j^{\prime}}+c_{j^{\prime}, k} c_{j, j^{\prime}}+c_{j^{\prime}, k} c_{i^{\prime}, j}, \\
& c_{k, i}=c_{k, k^{\prime}} c_{j^{\prime}, i}+c_{j^{\prime}, k} c_{k^{\prime}, i}=c_{k, k^{\prime}} c_{i, i^{\prime}}+c_{k, k^{\prime}} c_{k^{\prime}, i}+c_{j^{\prime}, k} c_{k^{\prime}, i},
\end{aligned}
$$

and hence $\Delta\left(\left(c_{k, k^{\prime}}, c_{j^{\prime}, k}, c_{i, i^{\prime}}, c_{k^{\prime}, i}, c_{j, j^{\prime}}, c_{i^{\prime}, j}\right)\right)=\left(c_{i, j}, c_{j, k}, c_{k, i}\right)$, as desired.

We now show a converse to Proposition 5.3, namely that every triple in the image of $S_{\geqslant 0}$ under the map $\Delta$ actually appears as labels of a triangle cut out of some classic Conway-Coxeter frieze. 
Proposition 5.4. Let $\left(a_{1}, a_{2}, b_{1}, b_{2}, c_{1}, c_{2}\right) \in S_{\geqslant 0}$. Then there exists a classic Conway-Coxeter frieze $\mathcal{C}=\left(c_{i, j}\right)$ and $i \leqslant j \leqslant k$ such that $\left(c_{i, j}, c_{j, k}, c_{k, i}\right)=$ $\Delta\left(\left(a_{1}, a_{2}, b_{1}, b_{2}, c_{1}, c_{2}\right)\right)$.

Proof. By Lemma 4.5, there are three classic Conway-Coxeter friezes $\tilde{\mathcal{C}}=\left(\tilde{c}_{i, j}\right)$, $\mathcal{C}^{\prime}=\left(c_{i, j}^{\prime}\right), \mathcal{C}^{\prime \prime}=\left(c_{i, j}^{\prime \prime}\right)$ such that

$$
\begin{aligned}
& \tilde{c}_{1,2}=1, \quad \tilde{c}_{2, \ell}=a_{1}, \quad \tilde{c}_{\ell, 1}=a_{2}, \\
& c_{1,2}^{\prime}=1, \quad c_{2, \ell^{\prime}}^{\prime}=b_{1}, \quad c_{\ell^{\prime}, 1}^{\prime}=b_{2}, \\
& c_{1,2}^{\prime \prime}=1, \quad c_{2, \ell^{\prime \prime}}^{\prime \prime}=c_{1}, \quad c_{\ell^{\prime \prime}, 1}^{\prime \prime}=c_{2}
\end{aligned}
$$

for some $\ell, \ell^{\prime}, \ell^{\prime \prime}$. Let $\tilde{\mathcal{T}}, \mathcal{T}^{\prime}, \mathcal{T}^{\prime \prime}$ be the corresponding triangulations of some regular polygons. We glue these three triangulations together in such a way that the edges with labels $\tilde{c}_{1,2}, c_{1,2}^{\prime}, c_{1,2}^{\prime \prime}$ become edges of an inner triangle and obtain a new triangulation $\mathcal{T}$. In the classic Conway-Coxeter frieze $\mathcal{C}$ corresponding to $\mathcal{T}$, this inner triangle carries labels $c_{i^{\prime}, j^{\prime}}=c_{j^{\prime}, k^{\prime}}=c_{k^{\prime}, i^{\prime}}=1$ for some $i^{\prime}, j^{\prime}, k^{\prime}$. Moreover, there are $i, j, k$ such that

$$
c_{i, i^{\prime}}=b_{1}, \quad c_{k^{\prime}, i}=b_{2}, \quad c_{j, j^{\prime}}=c_{1}, \quad c_{i^{\prime}, j}=c_{2}, \quad c_{k, k^{\prime}}=a_{1}, \quad c_{j^{\prime}, k}=a_{2} .
$$

In other words, our triangulation $\mathcal{T}$ has the shape as in Figure 7. Using the Ptolemy relations (Theorem 3.3) several times (as in the proof of Proposition 5.3), we obtain $\left(c_{i, j}, c_{j, k}, c_{k, i}\right)=\Delta\left(\left(a_{1}, a_{2}, b_{1}, b_{2}, c_{1}, c_{2}\right)\right)$.

Our goal is now to describe the image of $S_{\geqslant 0}=S \cap \mathbb{Z}_{\geqslant 0}^{6}$ under the map $\Delta: S \rightarrow$ $\mathbb{Z}^{3}$. The strategy will be the following. For a given triple $(a, b, c) \in \Delta\left(S_{\geqslant 0}\right) \subseteq \mathbb{Z}_{\geqslant 0}^{3}$, imagine that the preimage of $(a, b, c)$ under $\Delta$ is an iceberg. We are looking for a nonnegative preimage, that is, for a place on the iceberg that is not under water. In the first step, we find any place on the iceberg (Theorem 5.6); the second step (Theorem 5.9) is to move this solution (using Lemma 5.8) to the dry peak of the iceberg.

Theorem 5.10 clarifies the precise condition for $(a, b, c)$ to be in the image, Theorem 5.11 collects all these results and states the complete classification of triangles appearing in classic Conway-Coxeter friezes.

Definition 5.5. For a prime number $p$ and $n \in \mathbb{N}$, we denote by $v_{p}(n) \in \mathbb{Z}_{\geqslant 0}$ the exponent of $p$ in the prime factorization of $n$, that is, $p^{v_{p}(n)}$ divides $n$ but $p^{v_{p}(n)+1}$ does not divide $n$. We call $v_{p}(n)$ the $p$-valuation of $n$.

THEOREM 5.6. Let $a, b, c \in \mathbb{N}$ be such that

$$
d:=\operatorname{gcd}(a, b)=\operatorname{gcd}(b, c)=\operatorname{gcd}(a, c),
$$


and assume that either

$$
v_{2}(a)=v_{2}(b)=v_{2}(c)=0 \quad \text { or } \quad\left|\left\{v_{2}(a), v_{2}(b), v_{2}(c)\right\}\right|>1 .
$$

Then there exist $a_{1}, b_{2} \in \mathbb{Z}$ such that

$$
a_{1} a+b b_{2}=c \quad \text { and } \operatorname{gcd}\left(a_{1}, b\right)=\operatorname{gcd}\left(a, b_{2}\right)=1,
$$

that is, $\Delta\left(\left(a_{1}, b, a-b_{2}, b_{2}, 0,1\right)\right)=(a, b, c)$.

REMARK 5.7. Suppose $a, b, c$ satisfy the assumptions in Theorem 5.6. Then either all of $a, b, c$ are odd or we have $\left|\left\{v_{2}(a), v_{2}(b), v_{2}(c)\right\}\right|=2$ and the maximal value of $\left\{v_{2}(a), v_{2}(b), v_{2}(c)\right\}$ is attained only once. (In fact, for the second part, we use the condition on the greatest common divisors being equal.)

Proof. We set $a^{\prime}:=a / d, b^{\prime}:=b / d$ and $c^{\prime}:=c / d$. In particular, $a^{\prime}$ and $b^{\prime}$ are coprime, so we may choose $u, v \in \mathbb{Z}$ such that

$$
u a^{\prime}+v b^{\prime}=1 .
$$

Then multiplication by $c$ yields

$$
u c^{\prime} a+v c^{\prime} b=c .
$$

For any $k \in \mathbb{Z}$, set

$$
a_{1}:=u c^{\prime}+k b^{\prime}, \quad b_{2}:=v c^{\prime}-k a^{\prime} .
$$

Then

$$
a_{1} a+b b_{2}=\left(u c^{\prime}+k b^{\prime}\right) a+b\left(v c^{\prime}-k a^{\prime}\right)=c
$$

since $k b^{\prime} a=b k a^{\prime}$ by the definition of $a^{\prime}, b^{\prime}$.

It remains to find some $k \in \mathbb{Z}$ such that in addition, $\operatorname{gcd}\left(a_{1}, b\right)=\operatorname{gcd}\left(a, b_{2}\right)=1$.

We first claim that $\operatorname{gcd}\left(a_{1}, b^{\prime}\right) \mid c^{\prime}$; in fact, we have

$$
\operatorname{gcd}\left(a_{1}, b^{\prime}\right)=\operatorname{gcd}\left(u c^{\prime}+k b^{\prime}, b^{\prime}\right)=\operatorname{gcd}\left(u c^{\prime}, b^{\prime}\right) \mid u c^{\prime}
$$

and since $b^{\prime}$ and $u$ are coprime by (9), the claim follows.

Moreover, $b^{\prime}$ and $c^{\prime}$ are coprime by definition, so the above claim yields $\operatorname{gcd}\left(a_{1}, b^{\prime}\right)=1$ and we obtain

$$
\operatorname{gcd}\left(a_{1}, b\right)=\operatorname{gcd}\left(a_{1}, b^{\prime} d\right)=\operatorname{gcd}\left(a_{1}, d\right) .
$$

Similarly,

$$
\operatorname{gcd}\left(a, b_{2}\right)=\operatorname{gcd}\left(b_{2}, d\right)
$$


This means that it suffices to find suitable $k \in \mathbb{Z}$ such that $\operatorname{gcd}\left(a_{1}, d\right)=1=$ $\operatorname{gcd}\left(b_{2}, d\right)$.

If $d=1$, then we are clearly done. So assume $d>1$ and let $d=\prod_{i=1}^{r} p_{i}^{e_{i}}$ be the prime factorization (so $p_{i} \neq p_{j}$ for $i \neq j$ ). The idea now is to consider each prime divisor $p_{i}$ separately and find possible numbers $k_{i}$ such that $\operatorname{gcd}\left(a_{1}, p_{i}\right)=1=\operatorname{gcd}\left(b_{2}, p_{i}\right)$. This leads to different congruences and finally an overall suitable $k \in \mathbb{Z}$ such that $\operatorname{gcd}\left(a_{1}, p_{i}\right)=1=\operatorname{gcd}\left(b_{2}, p_{i}\right)$ for all $i=1, \ldots, r$ is constructed by using the Chinese remainder theorem.

Consider first an $i$ with $p_{i}>2$. If $p_{i}$ divides $b^{\prime}$, then $p_{i}$ does not divide $c^{\prime}$ (by definition) and $p_{i}$ does not divide $u$ by (9). Thus we have $\operatorname{gcd}\left(u c^{\prime}, p_{i}\right)=1$ and hence

$$
\operatorname{gcd}\left(a_{1}, p_{i}\right)=\operatorname{gcd}\left(u c^{\prime}+k b^{\prime}, p_{i}\right)=1
$$

for any $k$. If $p_{i}$ does not divide $b^{\prime}$, then $\operatorname{gcd}\left(a_{1}, p_{i}\right)=\operatorname{gcd}\left(u c^{\prime}+k b^{\prime}, p_{i}\right)=1$ for all but one $k \in\left\{0, \ldots, p_{i}-1\right\}$ (using that $b^{\prime}$ is invertible modulo $p_{i}$ ). Thus we get in all circumstances

$$
\left|\left\{k \in\left\{0, \ldots, p_{i}-1\right\} \mid \operatorname{gcd}\left(a_{1}, p_{i}\right)=1\right\}\right| \geqslant p_{i}-1 .
$$

It follows by symmetry that

$$
\left|\left\{k \in\left\{0, \ldots, p_{i}-1\right\} \mid \operatorname{gcd}\left(p_{i}, b_{2}\right)=1\right\}\right| \geqslant p_{i}-1 .
$$

But $2\left(p_{i}-1\right)>p_{i}$ by assumption; thus we find a $k_{i} \in\left\{0, \ldots, p_{i}-1\right\}$ such that $\operatorname{gcd}\left(a_{1}, p_{i}\right)=\operatorname{gcd}\left(p_{i}, b_{2}\right)=1$.

If $p_{i}=2$, then $d$ and hence all of $a, b, c$ are even. It follows from Remark 5.7 that precisely one of $a^{\prime}, b^{\prime}, c^{\prime}$ is even.

If $c^{\prime}$ is even, then $a^{\prime}, b^{\prime}$ are odd. But then $a_{1}$ and $b_{2}$ are both congruent to $k$ modulo 2; thus it suffices to choose any $k_{i}:=k$ odd.

The last case is when $a^{\prime}$ or $b^{\prime}$ is even. By symmetry, we can assume that $a^{\prime}$ is even (and hence $b^{\prime}$ and $c^{\prime}$ are odd). But then $a_{1} \equiv u+k(\bmod 2)$ and $b_{2} \equiv v \equiv$ $1(\bmod 2)$, where the last congruence follows from (9). Thus we may choose any $k_{i}:=k$ such that $u+k$ is odd.

Note that in all the above cases, the choice of $k_{i}$ is independent of adding multiples of $p_{i}$. So this yields a system of congruences

$$
k \equiv k_{i}\left(\bmod p_{i}\right) \quad \text { for } i=1, \ldots, r .
$$

The Chinese remainder theorem now gives a solution for $k$ as desired.

To understand the image of $\Delta$, we still need some more preparations. The following lemma describes a special element in a nice group of transformations leaving $\Delta$ invariant. 
LeMmA 5.8. Let $x:=\left(a_{1}, a_{2}, b_{1}, b_{2}, c_{1}, c_{2}\right) \in S, t \in \mathbb{Z}$ and

$\Gamma_{t}(x):=\left(a_{1} t-a_{2}, a_{1}(1-t)+a_{2},-b_{2}, b_{1}+b_{2}(t+1), c_{1} t+c_{2}(t-1), c_{1}+c_{2}\right)$.

Then $\Gamma_{t}(x) \in S$ and $\Delta(x)=\Delta\left(\Gamma_{t}(x)\right)$.

Proof. For the first claim, note that $\Gamma_{t}(x)$ is given by a block diagonal matrix with $2 \times 2$-matrices in $\mathrm{SL}_{2}(\mathbb{Z})$ on the diagonal. Therefore, elements in $S$ are mapped to elements in $S$. For the second claim, just evaluate $\Delta$.

THEOREM 5.9. Let $a, b, c \in \mathbb{N}$ be such that

$$
d:=\operatorname{gcd}(a, b)=\operatorname{gcd}(b, c)=\operatorname{gcd}(a, c),
$$

and assume that either

$$
v_{2}(a)=v_{2}(b)=v_{2}(c)=0 \quad \text { or } \quad\left|\left\{v_{2}(a), v_{2}(b), v_{2}(c)\right\}\right|>1 .
$$

Then there exists a tuple $\left(a_{1}, a_{2}, b_{1}, b_{2}, c_{1}, c_{2}\right) \in S \cap \mathbb{Z}_{\geqslant 0}^{6}$ such that

$$
\Delta\left(\left(a_{1}, a_{2}, b_{1}, b_{2}, c_{1}, c_{2}\right)\right)=(a, b, c) .
$$

Proof. By symmetry, we may assume that $c \leqslant a$ and $c \leqslant b$. Since we have the same assumptions as in Theorem 5.6, we get $a_{1}, b_{2} \in \mathbb{Z}$ such that $a_{1} a+b b_{2}=c$ and $\operatorname{gcd}\left(a_{1}, b\right)=\operatorname{gcd}\left(a, b_{2}\right)=1$. At least one of $a_{1}, b_{2}$ must be nonnegative (since $a, b, c \in \mathbb{N})$, so without loss of generality we can assume $a_{1} \geqslant 0$.

If $a_{1}=0$, then $b=1$ since $\operatorname{gcd}\left(a_{1}, b\right)=1$; but then $\Delta((0,1, a-c, c, 0,1))=$ $(a, b, c)$ and we are finished (note that $\operatorname{gcd}(a-c, c)=\operatorname{gcd}(a, c)=\operatorname{gcd}(a, b)=1)$. Thus assume that $a_{1}>0$ and let $a_{2}:=b, b_{1}:=a-b_{2}, c_{1}:=0$, and $c_{2}:=1$. Then $\Delta\left(\left(a_{1}, a_{2}, b_{1}, b_{2}, c_{1}, c_{2}\right)\right)=(a, b, c)$ and indeed $\left(a_{1}, a_{2}, b_{1}, b_{2}, c_{1}, c_{2}\right) \in S$. However, $b_{1}$ and $b_{2}$ may be negative in general, although $a_{1}, a_{2}, c_{1}, c_{2} \geqslant 0$.

We now show that applying the transformation $\Gamma_{t}$ of Lemma 5.8 several times eventually produces a tuple in $S \cap \mathbb{Z}_{\geqslant 0}^{6}$. In fact, let $t:=\left\lceil a_{2} / a_{1}\right\rceil \in \mathbb{N}$ and consider

$$
\Gamma_{t}\left(\left(a_{1}, a_{2}, b_{1}, b_{2}, c_{1}, c_{2}\right)\right)=:\left(\tilde{a}_{1}, \tilde{a}_{2}, \tilde{b}_{1}, \tilde{b}_{2}, \tilde{c}_{1}, \tilde{c}_{2}\right) \in S .
$$

Then by the definition of $t$, we have

$$
\begin{aligned}
& \tilde{a}_{1}=a_{1} t-a_{2} \geqslant 0, \\
& \tilde{a}_{2}=a_{1}(1-t)+a_{2}>0, \\
& \tilde{c}_{1}=c_{1} t+c_{2}(t-1)=t-1 \geqslant 0, \\
& \tilde{c}_{2}=c_{1}+c_{2}=1>0 .
\end{aligned}
$$


Moreover, we claim that $\tilde{b}_{1}=-b_{2} \geqslant 0$; in fact, $a_{1} a+b b_{2}=c \leqslant a$ and since $a_{1} a \geqslant a$, we conclude $b_{2} \leqslant 0$.

If $\tilde{b}_{2} \geqslant 0$ as well, then we are finished since $\left(\tilde{a}_{1}, \tilde{a}_{2}, \tilde{b}_{1}, \tilde{b}_{2}, \tilde{c}_{1}, \tilde{c}_{2}\right) \in S \cap \mathbb{Z}_{\geqslant 0}^{6}$ as desired. Otherwise, using that $b_{2} \leqslant 0$, we get

$$
\begin{aligned}
0 & >\tilde{b}_{2}=b_{1}+b_{2}(t+1)=b_{1}+b_{2}\left(\left\lceil a_{2} / a_{1}\right\rceil+1\right) \geqslant b_{1}+b_{2}\left(a_{2} / a_{1}+2\right) \\
& =\frac{1}{a_{1}}\left(a_{1} b_{1}+a_{2} b_{2}+2 a_{1} b_{2}\right)=\frac{c+a_{1} b_{2}}{a_{1}}=\frac{c}{a_{1}}+b_{2}>b_{2} .
\end{aligned}
$$

If $\tilde{a}_{1}=0$, then $\tilde{a}_{2}=1\left(\right.$ recall that $\left.\operatorname{gcd}\left(\tilde{a}_{1}, \tilde{a}_{2}\right)=1\right)$ and $c=\tilde{b}_{2} \geqslant 0$ (use the definition of $\Delta$ and Lemma 5.8) and we are finished. Thus, assume that $\tilde{a}_{1}>0$. But then we may replace $\left(a_{1}, a_{2}, b_{1}, b_{2}, c_{1}, c_{2}\right)$ by $\left(\tilde{a}_{1}, \tilde{a}_{2}, \tilde{b}_{1}, \tilde{b}_{2}, \tilde{c}_{1}, \tilde{c}_{2}\right)$ and repeat the same argument. Since $0>\tilde{b}_{2}>b_{2}$, by induction we will eventually obtain $\left(\tilde{a}_{1}, \tilde{a}_{2}, \tilde{b}_{1}, \tilde{b}_{2}, \tilde{c}_{1}, \tilde{c}_{2}\right) \in S \cap \mathbb{Z}_{\geqslant 0}^{6}$ as desired.

We now prove a converse to Theorem 5.9.

TheOREM 5.10. Let $a, b, c \in \mathbb{N}$ be such that $d:=\operatorname{gcd}(a, b)=\operatorname{gcd}(b, c)=$ $\operatorname{gcd}(a, c)$, and assume that there exists $\left(a_{1}, a_{2}, b_{1}, b_{2}, c_{1}, c_{2}\right) \in S \cap \mathbb{Z}_{\geqslant 0}^{6}$ such that

$$
\Delta\left(\left(a_{1}, a_{2}, b_{1}, b_{2}, c_{1}, c_{2}\right)\right)=(a, b, c) .
$$

Then either

$$
v_{2}(a)=v_{2}(b)=v_{2}(c)=0 \quad \text { or } \quad\left|\left\{v_{2}(a), v_{2}(b), v_{2}(c)\right\}\right|>1 .
$$

Proof. We prove the claim indirectly. Assume that $v_{2}(a)=v_{2}(b)=v_{2}(c)=\ell \geqslant 1$; thus we may write $a=2^{\ell} \tilde{a}, b=2^{\ell} \tilde{b}, c=2^{\ell} \tilde{c}$ with $\tilde{a}, \tilde{b}, \tilde{c}$ being odd. Let $\left(a_{1}, a_{2}, b_{1}, b_{2}, c_{1}, c_{2}\right) \in S \cap \mathbb{Z}_{\geqslant 0}^{6}$ be such that $\Delta\left(\left(a_{1}, a_{2}, b_{1}, b_{2}, c_{1}, c_{2}\right)\right)=(a, b, c)$. Then by Definition 5.2, we have

$$
\begin{aligned}
& a=b_{1}\left(c_{1}+c_{2}\right)+b_{2} c_{2}, \\
& b=a_{1} c_{1}+a_{2}\left(c_{1}+c_{2}\right), \\
& c=a_{1}\left(b_{1}+b_{2}\right)+a_{2} b_{2} .
\end{aligned}
$$

We claim that this implies

$$
\begin{array}{cc}
c_{1}+c_{2} \equiv b_{2} \equiv a_{1} \quad(\bmod 2) \\
c_{1} \equiv a_{2} \equiv b_{1}+b_{2} \quad(\bmod 2) .
\end{array}
$$

In fact, consider Equations (13)-(15). Since $a, b, c$ are even by assumption, the two summands on the right-hand side of each equation are both even or both odd. 
Suppose that in (13), both summands are even. The other case is dealt with similarly; the details are left to the reader. We distinguish two cases. If $b_{1}$ is even, then $b_{2}$ is odd (by the definition of $S$ ); hence $b_{1}+b_{2}$ is odd. Since $b_{2}$ is odd, our assumption on (13) implies that $c_{2}$ is even. Then $c_{1}$ is odd (again by the definition of $S$ ); thus $c_{1}+c_{2}$ is odd. But $a_{1}, a_{2}$ cannot both be even (by the definition of $S$ ), so (14) implies that $a_{1}$ and $a_{2}$ are both odd. This proves the claim on the above congruences in case $b_{1}$ is even. If $b_{1}$ is odd, then our assumption on (13) implies that $c_{1}+c_{2}$ is even. By the definition of $S$, we conclude that $c_{1}$ and $c_{2}$ are both odd. Then the assumption on (13) yields that $b_{2}$ is even. Then $b_{1}$ is odd (by the definition of $S$ ) and $b_{1}+b_{2}$ is odd. Now (14) implies that $a_{1}$ is even. So $a_{2}$ is odd (by the definition of $S$ ), and this completes the proof of the claim.

Hence, for the values of $a_{1}, a_{2}, b_{1}, b_{2}, c_{1}, c_{2}, c_{1}+c_{2}$ modulo 2 , only the following three cases are possible (recall that $b_{1}, b_{2}$ cannot both be even by the definition of $S$ ):

\begin{tabular}{c|c|c|c|c|c|c}
$b_{1}$ & $b_{2}$ & $a_{1}$ & $a_{2}$ & $c_{1}$ & $c_{2}$ & $c_{1}+c_{2}$ \\
\hline 0 & 1 & 1 & 1 & 1 & 0 & 1 \\
1 & 0 & 0 & 1 & 1 & 1 & 0 \\
1 & 1 & 1 & 0 & 0 & 1 & 1
\end{tabular}

By the definition of $S$, the numbers $b_{1}$ and $b_{2}$ are coprime, so we can choose $u, v \in \mathbb{Z}$ such that

$$
u b_{1}-v b_{2}=1
$$

Together with (13), this yields

$$
b_{1}\left(c_{1}+c_{2}\right)+b_{2} c_{2}=a=a u b_{1}-a v b_{2} ;
$$

hence $b_{1} \mid c_{2}+a v$ and $b_{2} \mid c_{1}+c_{2}-a u$ (using that $b_{1}$ and $b_{2}$ are coprime by the definition of $S$ ). So there exists $k_{1}, k_{2} \in \mathbb{Z}$ with

$$
c_{1}+c_{2}=a u-k_{1} b_{2}, \quad c_{2}=-a v+k_{2} b_{1} .
$$

Using Equations (13) and (16), $a=a+\left(k_{1}-k_{2}\right) b_{1} b_{2}$. Hence, we can choose $k_{1}=k_{2}$ (for the case $b_{1}=0$ or $b_{2}=0$, this is clear a priori). More precisely, from the possible parities in the above table, one sees that $k$ has to be odd in all circumstances.

Now we get from Equations (14) and (15) that

$$
\begin{aligned}
& b=a_{1}\left(a(u+v)-k\left(b_{1}+b_{2}\right)\right)+a_{2}\left(a u-k b_{2}\right), \\
& c=a_{1}\left(b_{1}+b_{2}\right)+a_{2} b_{2},
\end{aligned}
$$


which implies

$$
\begin{aligned}
b & =a\left(a_{1}(u+v)+a_{2} u\right)-k\left(a_{1}\left(b_{1}+b_{2}\right)+a_{2} b_{2}\right) \\
& =2^{\ell} \tilde{a}\left(a_{1}(u+v)+a_{2} u\right)-k 2^{\ell} \tilde{c} \\
& =2^{\ell}\left(\tilde{a}\left(a_{1}(u+v)+a_{2} u\right)-k \tilde{c}\right) .
\end{aligned}
$$

Thus because $\tilde{a}, \tilde{c}$ and $k$ are odd and $\tilde{b}=\tilde{a}\left(a_{1}(u+v)+a_{2} u\right)-k \tilde{c}$ is odd as well, we get that $a_{1}(u+v)+a_{2} u$ is even. Using this fact and the fact that $u, v$ are coprime by (16), the above table extends to:

\begin{tabular}{c|c|c|c|c|c}
$b_{1}$ & $b_{2}$ & $a_{1}$ & $a_{2}$ & $u$ & $v$ \\
\hline 0 & 1 & 1 & 1 & 1 & 0 \\
1 & 0 & 0 & 1 & 0 & 1 \\
1 & 1 & 1 & 0 & 1 & 1
\end{tabular}

But each of these three congruences for $b_{1}, b_{2}, u, v$ contradicts Equation (16).

As an immediate corollary to Theorems 5.9 and 5.10, we obtain the main result of this section, giving a complete classification of triples appearing as labels of triangles in classic Conway-Coxeter friezes.

THEOREM 5.11. Let $a, b, c \in \mathbb{N}$ be such that $d:=\operatorname{gcd}(a, b)=\operatorname{gcd}(b, c)=$ $\operatorname{gcd}(a, c)$. Then there exists $\left(a_{1}, a_{2}, b_{1}, b_{2}, c_{1}, c_{2}\right) \in S \cap \mathbb{Z}_{\geqslant 0}^{6}$ such that

$$
\Delta\left(\left(a_{1}, a_{2}, b_{1}, b_{2}, c_{1}, c_{2}\right)\right)=(a, b, c)
$$

if and only if either

$$
\nu_{2}(a)=v_{2}(b)=v_{2}(c)=0 \quad \text { or } \quad\left|\left\{v_{2}(a), v_{2}(b), v_{2}(c)\right\}\right|>1 .
$$

THEOREM 5.12. Let $a, b, c \in \mathbb{N}$. Then the triple $(a, b, c)$ appears as labels of $a$ triangle in a classic Conway-Coxeter frieze if and only if $\operatorname{gcd}(a, b)=\operatorname{gcd}(b, c)$ $=\operatorname{gcd}(a, c)$ and

$$
v_{2}(a)=v_{2}(b)=v_{2}(c)=0 \quad \text { or } \quad\left|\left\{v_{2}(a), v_{2}(b), v_{2}(c)\right\}\right|>1 .
$$

Proof. By Propositions 5.3 and 5.4 and Lemma 4.3, a triple $(a, b, c)$ appears as labels of a triangle in a classic Conway-Coxeter frieze if and only if $\operatorname{gcd}(a, b)=$ $\operatorname{gcd}(b, c)=\operatorname{gcd}(a, c)$ and if there exists a tuple $\left(a_{1}, a_{2}, b_{1}, b_{2}, c_{1}, c_{2}\right) \in S \cap \mathbb{Z}_{\geqslant 0}^{6}$ such that

$$
\Delta\left(\left(a_{1}, a_{2}, b_{1}, b_{2}, c_{1}, c_{2}\right)\right)=(a, b, c) .
$$

The claim now follows by Theorem 5.11. 


\section{Finiteness}

In this section, we prove finiteness results on the number of possible frieze patterns with coefficients over subsets $R \subseteq \mathbb{C}$. Our main interest is in frieze patterns with coefficients over $\mathbb{N}$, but it will turn out that our finiteness result holds for arbitrary subsets $R$ of $\mathbb{C}$ without an accumulation point in $\mathbb{C}$.

The following result is the key step. It is a generalization of an analogous result for classic frieze patterns; see [7, Theorem 3.6].

We recall some standard notation [3]. For a frieze pattern with coefficients $\mathcal{C}=\left(c_{i, j}\right)$ of height $n$ as in Definition 2.1, we call the sequence $\left(c_{i, i+2}\right)_{i \in \mathbb{Z}}$ the quiddity cycle of $\mathcal{C}$. Moreover, for a tame frieze pattern we have $c_{i, i+2}=$ $c_{n+i+3, n+i+5}$ by glide symmetry (see Theorem 2.12 ); hence the quiddity cycle is completely determined by any (consecutive) subsequence of length $n+3$.

LEMMA 6.1. Let $R \subseteq \mathbb{C} \backslash\{0\}$ be a subset such that

$$
M:=\inf \{|x|: x \in R\}>0 .
$$

Let $\mathcal{C}=\left(c_{i, j}\right)$ be a frieze pattern with coefficients of height $n \geqslant 1$ over $R$. Consider the boundary sequence $\left(c_{0,1}, c_{1,2}, \ldots, c_{n+2, n+3}\right)$ and set

$$
P:=\max \left\{\left|c_{i, i+1}\right|: 0 \leqslant i \leqslant n+2\right\} .
$$

If $P \geqslant 1$, then every entry in the quiddity cycle of $\mathcal{C}$ has absolute value at most

$$
\frac{P^{2}\left(P M+(n-1) P^{2}+M\right)}{M^{2}} .
$$

Proof. We set $B:=P^{2}\left(P M+(n-1) P^{2}+M\right) / M^{2}$ for brevity. Note that $B>0$ since $P>0$ and $M>0$ by assumption. To ease notation, we also set $d_{i}:=c_{i, i+1}$ for all $i \in \mathbb{Z}$.

Suppose for a contradiction that there exists an entry $x_{1}$ in the quiddity cycle such that $\left|x_{1}\right|>B$. Then we consider the corresponding two consecutive rows in the frieze pattern $\mathcal{C}$, with notation as in the following figure:

$$
\begin{array}{cccccccccc}
0 & d_{j} & x_{1} & x_{2} & \ldots & x_{n-1} & x_{n} & d_{j-1} & 0 & \\
& 0 & d_{j+1} & y_{1} & y_{2} & \ldots & y_{n-1} & y_{n} & d_{j} & 0 .
\end{array}
$$

Note that the boundaries are as indicated because of the glide symmetry on the boundary entries; see Remark 2.2.

The strategy of the proof is to proceed inductively along the rows. More precisely, we want to use the following two conditions as induction hypotheses: 
(1) $\left|x_{k}\right|>\left(\left(M\left(M B-P^{2}\right) / P^{2}\right)-(k-2) P^{2}\right)(1 / M)$ for $k=2, \ldots, i+1$,

(2) $\left|y_{k}\right|<\left(M P^{2} /\left(M\left(M B-P^{2}\right)-(k-2) P^{4}\right)\right)\left(P^{2}+M\left|x_{k+1}\right|\right)$ for $k=2, \ldots, i$.

For the base of the induction, let us check that condition (1) holds for $k=2$ and $k=3$ and that condition (2) holds for $k=2$.

By the defining condition for frieze patterns with coefficients, the triangle inequality, the definition of $P$ and our assumption that $P \geqslant 1$, we have $\left|x_{1} y_{1}\right|=\left|d_{j} d_{j+2}+d_{j+1} x_{2}\right| \leqslant\left|d_{j} d_{j+2}\right|+\left|d_{j+1} x_{2}\right| \leqslant P^{2}+P\left|x_{2}\right| \leqslant P^{2}\left(1+\left|x_{2}\right|\right)$.

Using the definition of $M$ and the assumption $\left|x_{1}\right|>B$ this yields

$$
M \leqslant\left|y_{1}\right| \leqslant \frac{P^{2}\left(1+\left|x_{2}\right|\right)}{\left|x_{1}\right|}<\frac{P^{2}\left(1+\left|x_{2}\right|\right)}{B} .
$$

Solving for $\left|x_{2}\right|$, we obtain

$$
\left|x_{2}\right|>\frac{M B}{P^{2}}-1=\frac{M B-P^{2}}{P^{2}},
$$

which is condition (1) for $k=2$.

Going one step to the right, the defining condition for frieze patterns with coefficients reads as

$$
x_{2} y_{2}-x_{3} y_{1}=d_{j} d_{j+3} .
$$

By similar arguments as above and by using Equations (17) and (18), we conclude

$$
\begin{aligned}
M & \leqslant\left|y_{2}\right| \leqslant \frac{\left|d_{j} d_{j+3}\right|+\left|x_{3} y_{1}\right|}{\left|x_{2}\right|} \leqslant P^{2} \frac{B+\left|x_{3}\right|+\left|x_{3}\right| \cdot\left|x_{2}\right|}{B\left|x_{2}\right|} \\
& =P^{2}\left(\frac{1}{\left|x_{2}\right|}+\frac{\left|x_{3}\right|}{B}\left(\frac{1}{\left|x_{2}\right|}+1\right)\right) \\
& <P^{2}\left(\frac{P^{2}}{M B-P^{2}}+\frac{\left|x_{3}\right|}{B}\left(\frac{P^{2}}{M B-P^{2}}+1\right)\right) \\
& =\frac{P^{2}}{M B-P^{2}}\left(P^{2}+M\left|x_{3}\right|\right) .
\end{aligned}
$$

This shows Equation (2) for $k=2$.

Solving this inequality for $\left|x_{3}\right|$ then gives

$$
\left|x_{3}\right|>\left(\frac{M\left(M B-P^{2}\right)}{P^{2}}-P^{2}\right) \frac{1}{M}
$$

and this is Equation (1) for $k=3$. 
Thus we have shown that we can indeed use conditions (1) and (2) as induction hypotheses for an induction on $i$.

For the induction step, we have to show that condition (2) holds for $i+1$ and that condition (1) holds for $i+2$.

From the defining condition of frieze patterns with coefficients, we have

$$
x_{i+1} y_{i+1}-x_{i+2} y_{i}=d_{j} d_{j+i+2}
$$

and hence

$$
\left|y_{i+1}\right| \leqslant \frac{\left|d_{j} d_{j+i+2}\right|+\left|x_{i+2}\right| \cdot\left|y_{i}\right|}{\left|x_{i+1}\right|} \leqslant \frac{P^{2}+\left|x_{i+2}\right| \cdot\left|y_{i}\right|}{\left|x_{i+1}\right|} .
$$

Using the induction hypothesis for $\left|y_{i}\right|$, we deduce

$$
\left|y_{i+1}\right|<\frac{P^{2}}{\left|x_{i+1}\right|}+\frac{M P^{2}\left|x_{i+2}\right|}{M\left(M B-P^{2}\right)-(i-2) P^{4}}\left(\frac{P^{2}}{\left|x_{i+1}\right|}+M\right) .
$$

Now we also plug in the induction hypothesis for $\left|x_{i+1}\right|$ and get

$$
\begin{aligned}
\left|y_{i+1}\right|< & \frac{P^{4} M}{M\left(M B-P^{2}\right)-(i-1) P^{4}} \\
& +\frac{M P^{2}\left|x_{i+2}\right|}{M\left(M B-P^{2}\right)-(i-2) P^{4}}\left(\frac{P^{4} M}{M\left(M B-P^{2}\right)-(i-1) P^{4}}+M\right) \\
= & \frac{M P^{2}}{M\left(M B-P^{2}\right)-(i-1) P^{4}}\left(P^{2}+M\left|x_{i+2}\right|\right) .
\end{aligned}
$$

This proves the induction step for $\left|y_{i+1}\right|$.

Now we solve the last inequality for $\left|x_{i+2}\right|$. Since $M \leqslant\left|y_{i+1}\right|$, this yields

$$
\left|x_{i+2}\right|>\left(\frac{M\left(M B-P^{2}\right)-(i-1) P^{4}}{P^{2}}-P^{2}\right) \frac{1}{M}=\left(\frac{M\left(M B-P^{2}\right)}{P^{2}}-i P^{2}\right) \frac{1}{M}
$$

and this proves the inductive step for $\left|x_{i+2}\right|$.

Noting that $\left|x_{n+1}\right|=\left|d_{j-1}\right| \leqslant P$, our induction argument eventually yields

$$
\left|y_{n}\right|<\frac{M P^{3}}{M\left(M B-P^{2}\right)-(n-2) P^{4}}(P+M) .
$$

On the other hand, by the definition of $B$, we have

$$
M\left(M B-P^{2}\right)-(n-2) P^{4}=P^{3} M+(n-1) P^{4}-(n-2) P^{4}=P^{3}(M+P) .
$$

Together with Equation (19) this implies $\left|y_{n}\right|<M$, a contradiction to the definition of $M$ and the fact that the frieze pattern $\mathcal{C}$ has nonzero entries by assumption. This means that the assumption $\left|x_{1}\right|>B$ was wrong and hence every entry in the quiddity cycle of $\mathcal{C}$ has absolute value at most $B$, as claimed. 
REMARK 6.2. Lemma 6.1 does not hold without the assumption $P \geqslant 1$. As an example, consider the following frieze pattern with coefficients

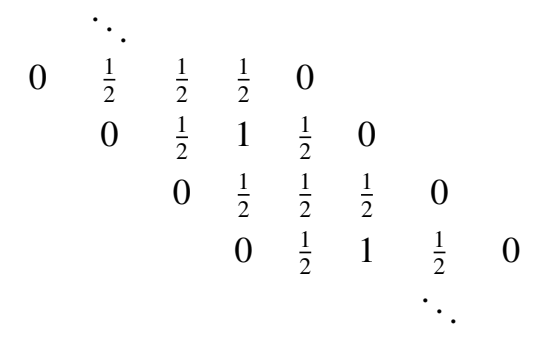

This is obtained from a classic Conway-Coxeter frieze pattern of height $n=1$ by scaling with the factor $\frac{1}{2}$; see Remark 2.3. With the notation of Lemma 6.1 we have $R=\frac{1}{2} \mathbb{N}$, the positive half-integers, and $M=\frac{1}{2}=P$. Then the bound given in Lemma 6.1 is equal to $\frac{3}{4}$, which is clearly not an upper bound for all entries in the quiddity cycle.

As a consequence of Lemma 6.1, we obtain the following finiteness result for frieze patterns with coefficients over subsets of complex numbers without accumulation points. This generalizes [7, Corollary 3.8] from classic frieze patterns to frieze patterns with coefficients.

Proposition 6.3. Let $R \subseteq \mathbb{C} \backslash\{0\}$ be a subset without accumulation point in $\mathbb{C}$. Let $n \in \mathbb{Z}_{\geqslant 0}$ and fix a boundary sequence in $R^{n+3}$. Then there are only finitely many frieze patterns with coefficients of height $n$ over $R$ with the given boundary sequence.

Proof. For $n=0$, there is only one possible frieze pattern with coefficients for any given boundary sequence; see Example 2.4.

For $n \geqslant 1$, we want to use Lemma 6.1. Recall that frieze patterns with coefficients can be scaled (see Remark 2.3). The existence of only finitely many frieze patterns with coefficients over $R$ and the property of $R$ not having an accumulation point are invariant under scaling, so we can assume that our boundary sequence satisfies the assumption $P \geqslant 1$ in Lemma 6.1.

By assumption, the origin is not an accumulation point and $R$ satisfies the assumption of Lemma 6.1. Moreover, again by assumption, every closed disk in $\mathbb{C}$ contains only finitely many elements from $R$. Then Lemma 6.1 implies that there are only finitely many elements of $R$, which can appear in the quiddity cycle of a frieze pattern with coefficients having the given boundary sequence. However, any nonzero frieze pattern with coefficients is determined by the boundary sequence and the quiddity cycle, and this proves the claim. 


\section{Acknowledgements}

We are grateful to the anonymous referees for a careful reading of the paper and for numerous useful suggestions. The publication of this article was funded by the Open Access Fund of the Leibniz Universität Hannover.

\section{Conflict of Interest: None.}

\section{References}

[1] K. Baur, E. Faber, S. Gratz, K. Serhiyenko and G. Todorov, 'Friezes satisfying higher $\mathrm{SL}_{k}$-determinants', Preprint, 2018, arXiv:1810.10562.

[2] D. Broline, D. W. Crowe and I. M. Isaacs, 'The geometry of frieze patterns', Geom. Dedicata 3 (1974), 171-176.

[3] J. H. Conway and H. S. M. Coxeter, 'Triangulated polygons and frieze patterns', Math. Gaz. 57 (1973), no. 400, 87-94 and no. 401, 175-183.

[4] H. S. M. Coxeter, 'Frieze patterns', Acta Arith. 18 (1971), 297-310.

[5] M. Cuntz, 'On wild frieze patterns', Exp. Math. 26 (2017), 342-348.

[6] M. Cuntz, 'A combinatorial model for tame frieze patterns', Münster J. Math. 12(1) (2019), 49-56.

[7] M. Cuntz and T. Holm, 'Frieze patterns over integers and other subsets of the complex numbers', J. Comb. Algebra 3 (2019), 153-188.

[8] S. Fomin and A. Zelevinsky, 'Cluster algebras I: foundations', J. Amer. Math. Soc. 15 (2002), 497-529.

[9] S. Fomin and A. Zelevinsky, ' $Y$-systems and generalized associahedra', Ann. of Math. (2) 158(3) (2003), 977-1018.

[10] T. Holm and P. Jørgensen, 'A p-angulated generalisation of Conway and Coxeter's theorem on frieze patterns', Int. Math. Res. Not. 2020(1) (2020), 71-90.

[11] S. Morier-Genoud, 'Coxeter's frieze patterns at the crossroads of algebra, geometry and combinatorics', Bull. Lond. Math. Soc. 47(6) (2015), 895-938.

[12] S. Morier-Genoud and V. Ovsienko, 'Farey boat: continued fractions and triangulations, modular group and polygon dissections', Jahresber. Dtsch. Math.-Ver. 121(2) (2019), 91-136.

[13] J. Propp, 'The combinatorics of frieze patterns and Markoff numbers', Preprint, 2005, arXiv: math/0511633.

[14] R. Schiffler, 'A cluster expansion formula ( $A_{n}$ case)', Electron. J. Combin. 15(1) (2008), Research paper 64, 9 pages.

[15] D. E. Speyer, 'Perfect matchings and the octahedron recurrence', J. Algebraic Combin. 25(3) (2007), 309-348.

[16] J. J. Sylvester, 'On the relation between the minor determinants of linearly equivalent quadratic functions', Philos. Mag., s. IV I (1851), 295-305. 